\title{
EXPONENTIAL APPROXIMATIONS FOR THE PRIMITIVE EQUATIONS OF THE OCEAN
}

\author{
R. TEMAM AND D. WIROSOETISNO
}

\begin{abstract}
We show that in the limit of small Rossby number $\varepsilon$, the primitive equations of the ocean (OPEs) can be approximated by "higher-order quasigeostrophic equations" up to an exponential accuracy in $\varepsilon$. This approximation assumes well-prepared initial data and is valid for a timescale of order one (independent of $\varepsilon$ ). Our construction uses Gevrey regularity of the OPEs and a classical method to bound errors in higher-order perturbation theory.
\end{abstract}

\section{INTRODUCTION}

We consider the primitive equations for the ocean (henceforth OPEs), scaled as in 13

$$
\begin{aligned}
& \partial_{t} \boldsymbol{v}+\frac{1}{\varepsilon}\left[\boldsymbol{v}^{\perp}+\nabla p\right]+\boldsymbol{u} \cdot \boldsymbol{\nabla} \boldsymbol{v}=\mu \Delta_{3} \boldsymbol{v}+f_{\boldsymbol{v}}, \\
& \partial_{t} \rho-\frac{1}{\varepsilon} w+\boldsymbol{u} \cdot \boldsymbol{\nabla} \rho=\mu \Delta_{3} \rho+f_{\rho}, \\
& \boldsymbol{\nabla} \cdot \boldsymbol{u}=\boldsymbol{\nabla} \cdot \boldsymbol{v}+w_{z}=0, \\
& \rho=-p_{z} .
\end{aligned}
$$

Here $\boldsymbol{u}=(u, v, w)$ is the three-dimensional fluid velocity, with $\boldsymbol{v}=(u, v)$ its horizontal component and $\boldsymbol{v}^{\perp}=(-v, u) ; p$ is the pressure; $\rho$ is the perturbation density (not including the mean stable stratification which figures into the $\varepsilon$ in the equation for $\rho)$. We write $\nabla_{2}:=\left(\partial_{x}, \partial_{y}\right)$ and $\nabla_{3}:=\left(\partial_{x}, \partial_{y}, \partial_{z}\right)$; when no ambiguity may arise, we simply write $\nabla$. Similarly, we write $\Delta_{2}:=\partial_{x}^{2}+\partial_{y}^{2}$ and $\Delta_{3}:=\partial_{x}^{2}+\partial_{y}^{2}+\partial_{z}^{2}$. The parameter $\varepsilon$ is related to the Rossby and Froude numbers; in this article we shall be concerned with the limit $\varepsilon \rightarrow 0$, and for convenience we assume that $\varepsilon \leq 1$ (further restrictions on $\varepsilon$ will be stated below). In general the viscosity coefficients for $\boldsymbol{v}$ and $\rho$ are different; we have set them all to $\mu$ for clarity of presentation (the general case does not introduce any more essential difficulty). The forcings $f_{\boldsymbol{v}}$ and $f_{\rho}$ are assumed to be independent of time.

We work in three spatial dimensions, $\boldsymbol{x}=(x, y, z) \in\left[0, L_{1}\right] \times\left[0, L_{2}\right] \times\left[-L_{3} / 2, L_{3} / 2\right]$ $=: \mathcal{M}$, with periodic boundary conditions assumed. Following common practice in

2000 Mathematics Subject Classification. Primary: 35B25, 76 U05.

Key words and phrases. Singular perturbation, exponential asymptotics, Gevrey regularity, primitive equations.

This research was supported by grants NSF 0305110, DOE DE-FG02-01ER63251:A000 and the Research Fund of Indiana University. 
numerical simulations of stratified turbulence (see, e.g., [2]), the dependent variables are assumed to have the following symmetries:

$$
\begin{array}{llrl}
\boldsymbol{v}(x, y,-z) & =\boldsymbol{v}(x, y, z), & & p(x, y,-z)=p(x, y, z), \\
w(x, y,-z) & =-w(x, y, z), & & \rho(x, y,-z)=-\rho(x, y, z) ;
\end{array}
$$

we say that $\boldsymbol{v}$ and $p$ are even in $z$, while $w$ and $\rho$ are odd in $z$. If in addition $f_{\boldsymbol{v}}$ is even and $f_{\rho}$ is odd in $z$, it can be verified that this symmetry is preserved by the OPEs (1.1), that is, if it holds at $t=0$, it continues to hold for $t>0$. Since $w$ and $p$ are periodic in $z$, we have $w\left(x, y,-L_{3} / 2\right)=w\left(x, y, L_{3} / 2\right)=0$ and $\rho\left(x, y,-L_{3} / 2\right)=\rho\left(x, y, L_{3} / 2\right)=0$; similarly, $u_{z}=0, v_{z}=0$ and $p_{z}=0$ on $z=0, \pm L_{3} / 2$ if they are sufficiently smooth (as will be assumed below). One may consider the symmetry conditions (1.2) as a way to impose the boundary conditions $w=0, \rho=0, u_{z}=0, v_{z}=0$ and $p_{z}=0$ on both $z=0$ and $z=L_{3} / 2$, in the effective domain $\left[0, L_{1}\right] \times\left[0, L_{2}\right] \times\left[0, L_{3} / 2\right]$. All variables and the forcing are assumed to have zero mean over $\mathcal{M}$; the symmetry conditions above ensure that this also holds for their products that appear below.

It is known that, given sufficiently regular initial data, the OPEs have a unique strong solution for all time [4, [7]; the results of [14 then imply that a very regular solution, belonging to a Gevrey space defined below, exists for all time. The existence of finite-dimensional global attractors in various spaces (see [15]), and thus uniform boundedness of the solution, have been shown in [6]; see also the result in 12. One can thus regard the OPEs as equivalent to a finite-dimensional system. This fact is an important ingredient for our main result.

As noted earlier, we are concerned with the limit $\varepsilon \rightarrow 0$. A flow is said to be in geostrophic balance if the $\mathcal{O}(1 / \varepsilon)$ terms in $(1.1$ ) $)$ vanish, i.e. if

$$
\boldsymbol{v}=\nabla^{\perp} p
$$

where $\boldsymbol{\nabla}^{\perp}:=\left(-\partial_{y}, \partial_{x}\right)$. Note that this implies $\boldsymbol{\nabla}^{\perp} \cdot \boldsymbol{v}=0$, which with the fact that $w$ is odd in $z$ in turn implies that $w=0$, so the $\mathcal{O}(1 / \varepsilon)$ term in (1.1p) also vanishes. Assuming that (1.3) is satisfied initially, it is well known that the OPEs can be approximated by the simpler quasi-geostrophic equation (QGE),

$$
\partial_{t} q^{\mathrm{g}}+\boldsymbol{v}^{\mathrm{g}} \cdot \nabla q^{\mathrm{g}}=\mu \Delta_{3} q^{\mathrm{g}}+f_{q},
$$

which only involves a single variable $q^{\mathrm{g}}$; here $f_{q}:=\nabla^{\perp} \cdot f_{\boldsymbol{v}}-\partial_{z} f_{\rho}$. The original variables are recovered using

$$
\boldsymbol{v}^{\mathrm{g}}=\nabla^{\perp} \Delta_{3}^{-1} q^{\mathrm{g}} \quad \text { and } \quad \rho^{\mathrm{g}}=-\partial_{z} \Delta_{3}^{-1} q^{\mathrm{g}},
$$

Here $\Delta_{3}^{-1}$ is uniquely defined to have zero mean over $\mathcal{M}$. The quantities $\boldsymbol{v}^{\mathrm{g}}$ and $\rho^{\mathrm{g}}$ derived from the potential vorticity $q^{\mathrm{g}}$ are said to be geostrophic. The fact that $\partial_{z} w+\boldsymbol{\nabla} \cdot \boldsymbol{v}=0$ and (1.5) imply that $w^{\mathrm{g}}=0$.

An approximation result was obtained in [3] for a closely related system where it was shown that the QGE (1.4) with $\mu=0$ and $f_{q}=0$ approximates (the unforced, inviscid and Boussinesq analogue of) the OPE (1.1) with an error of order $\varepsilon$ for $t \in[0, T]$, viz.,

$$
\left|\boldsymbol{v}(t)-\nabla^{\perp} \Delta_{3}^{-1} q^{\mathrm{g}}(t)\right|_{H^{4}(\mathcal{M})}+|w(t)|_{H^{4}(\mathcal{M})}+\left|\rho(t)+\partial_{z} \Delta_{3}^{-1} q^{\mathrm{g}}(t)\right|_{H^{4}(\mathcal{M})} \leq \varepsilon
$$

assuming that the left-hand side less than $\varepsilon$ at $t=0$ (and given sufficient regularity). We shall not attempt to follow their approach in this article as it makes no qualitative difference for our exponential-order result. 
Regarding this result as a first-order approximation in the parameter $\varepsilon$, a natural question is whether one can obtain higher-order approximations. Put differently, we would like to know how fast, as $\varepsilon \rightarrow 0$, the solution of the PE converges to the solution of a simpler system analogous to the QGE (1.4). Our main purpose in this article is to show that convergence of any algebraic order is possible for $\varepsilon$ sufficiently small, resulting in an error estimate which is exponentially small in $\varepsilon$.

In the geophysical parlance, the dynamics of a fluid flow is said to be balanced if the solution stays near a subspace ("balance manifold") for some time. The order of the balance dynamics measures how close this approximation is in terms of $\varepsilon$. A result such as (1.6) implies that the quasi-geostrophic relations (1.5) define a balance dynamics of order $\varepsilon$. Our result (Theorem 1 below) thus implies that a balanced dynamics of exponential order exists for a timescale of order one (i.e. independent of the Rossby number). Note, however, that our result is actually stronger than the geophysical definition of balance, since it gives a pointwise approximation to an exponential order.

Also of geophysical interest is whether the solution (when appropriately initialised) stays close to a balance manifold over longer timescales. Using careful estimates (of a different type than those used here), it may be possible to prove that the solution remains "exponentially balanced" for timescales of order $1 / \varepsilon$ (although it is clear that one cannot expect pointwise accuracy over this timescale); we plan to report on this in a future work [16].

We begin with some notations. We write the horizontal velocity $\boldsymbol{v}$ as

$$
\boldsymbol{v}(x, y, z)=\overline{\boldsymbol{v}}(z)+\nabla^{\perp} \psi+\nabla \chi
$$

where $\overline{\boldsymbol{v}}(z)$ is the mean vertical shear, i.e. the $(x, y)$-average of $\boldsymbol{v}$. We introduce the linearised potential vorticity (which has appeared in the QGE above)

$$
q:=\nabla^{\perp} \cdot \boldsymbol{v}-\rho_{z} .
$$

The streamfunction $\psi$ and velocity potential $\chi$ are defined as follows. First, let

$$
\chi:=\Delta_{2}^{-1} \nabla \cdot \boldsymbol{v}
$$

where, here and henceforth, $\Delta_{2}^{-1}$ is uniquely defined to have zero mean on each $(x, y)$-plane. Next, let

$$
\phi(x, y, z):=\int_{0}^{z} \Delta_{2}^{-1}\left[\Delta_{3} \rho+\partial_{z^{\prime}} q\right] \mathrm{d} z^{\prime}
$$

And finally, let

$$
\psi:=\Delta_{3}^{-1}\left[q+\phi_{z z}\right]
$$

where $\Delta_{3}^{-1}$ is uniquely defined to have zero average over $\mathcal{M}$. This completes the definition of $(q, \overline{\boldsymbol{v}}, \chi, \phi)$ in terms of $(\boldsymbol{v}, \rho)$.

It can be verified that these definitions imply the following relations,

$$
\Delta_{2} \psi=\nabla^{\perp} \cdot \boldsymbol{v}, \quad p=\psi-\phi \quad \text { and } \quad \Delta_{2} \chi=\nabla \cdot \boldsymbol{v} .
$$

The second equation is useful to compute $\rho=-\partial_{z} p$ from $q$ and $\phi ; \boldsymbol{v}$ can be computed from $(q, \overline{\boldsymbol{v}}, \chi, \phi)$ using (1.7) and (1.11). Using the incompressibility condition $\boldsymbol{\nabla} \cdot \boldsymbol{v}+w_{z}=0$ and the fact that $w(x, y, 0)=0$, we also have

$$
w(x, y, z)=-\int_{0}^{z} \Delta_{2} \chi\left(x, y, z^{\prime}\right) \mathrm{d} z^{\prime} .
$$


The primitive equations (1.1) can now be written in the following form,

$$
\begin{array}{rcc}
\partial_{t} q & +\nabla^{\perp} \cdot(\boldsymbol{u} \cdot \nabla \boldsymbol{v})-\partial_{z}(\boldsymbol{u} \cdot \boldsymbol{\nabla} \rho) & =\mu \Delta_{3} q+f_{q} \\
\partial_{t} \overline{\boldsymbol{v}}_{z}+\frac{1}{\varepsilon} \overline{\boldsymbol{v}}_{z}^{\perp}+\partial_{z}\left(\overline{w \boldsymbol{v}_{z}}\right) & =\mu \overline{\boldsymbol{v}}_{z z z}+\partial_{z} \bar{f}_{\boldsymbol{v}} \\
\partial_{t} \Delta_{3} \chi-\frac{1}{\varepsilon} \Delta_{3} \phi+\Delta_{3} \Delta_{2}^{-1} \boldsymbol{\nabla} \cdot(\boldsymbol{u} \cdot \boldsymbol{\nabla} \boldsymbol{v}) & =\mu \Delta_{3}^{2} \chi+\Delta_{3} f_{\chi} \\
\partial_{t} \phi_{z z}+\frac{1}{\varepsilon} \Delta_{3} \chi+\partial_{z z} \Delta_{2}^{-1} \nabla^{\perp} \cdot(\boldsymbol{u} \cdot \boldsymbol{\nabla} \boldsymbol{v})+\mathrm{P}_{z} \partial_{z}(\boldsymbol{u} \cdot \boldsymbol{\nabla} \rho) & =\mu \Delta_{3} \phi_{z z}+\partial_{z z} f_{\phi},
\end{array}
$$

where overbar denotes average over $(x, y)$ and $\mathrm{P}_{z}$ is a projection which removes the $(x, y)$-average of a function, $\mathrm{P}_{z} \rho:=\rho-\bar{\rho}$, etc. The forcing terms in (1.14) are related to those in (1.1) by

$$
\begin{array}{ll}
f_{q}=\nabla^{\perp} \cdot f_{\boldsymbol{v}}-\partial_{z} f_{\rho}, & f_{\chi}=\Delta_{2}^{-1} \nabla \cdot f_{\boldsymbol{v}}, \\
\bar{f}_{\boldsymbol{v}}=\overline{f_{\boldsymbol{v}}}, & \partial_{z z} f_{\phi}=\Delta_{2}^{-1} \partial_{z z} \nabla^{\perp} \cdot f_{\boldsymbol{v}}+\mathrm{P}_{z} \partial_{z} f_{\rho} .
\end{array}
$$

It is easily verified that the $\mathcal{O}(1 / \varepsilon)$ terms in (1.14) are antisymmetric. If the fast variables $(\overline{\boldsymbol{v}}, \chi, \phi)$ are all zero - a condition that is equivalent to the geostrophic balance relation (1.3) - they can only grow through the nonlinear terms involving the slow variable $q$ and the nonlinear terms on the right-hand side. Further analysis (cf. [3], 16]) shows that they remain small over a timescale of order one. Our main result [Theorem 1] states that, for certain initial data $(\overline{\boldsymbol{v}}, \chi$ and $\phi$ given in terms of $q$ ), this system can be approximated up to an error exponentially small in $\varepsilon$ by the solution of an equation for a finite-dimensional slow variable $\tilde{q}$.

We note that the form (1.14) will only be used for (i) the construction of the approximation $\boldsymbol{v}^{*}(\tilde{q} ; \varepsilon)$ and $\rho^{*}(\tilde{q} ; \varepsilon)$, and (ii) the integration of the finite-dimensional variable $\tilde{q}$. The approximation error estimates are in terms of $(\boldsymbol{v}, \rho)$ and $\left(\boldsymbol{v}^{*}, \rho^{*}\right)$, so we will not need a priori estimates for (1.14).

\section{Gevrey Regularity and Statement of the Main Result}

Let $W=(u, v, \rho)$. The periodic boundary conditions allow us to write functions (assuming sufficient regularity) in Fourier series,

$$
W(\boldsymbol{x}, t)=\sum_{\boldsymbol{k} \in \mathbb{Z}^{3}} W_{\boldsymbol{k}}(t) \mathrm{e}^{\mathrm{i} \boldsymbol{k}^{\prime} \cdot \boldsymbol{x}},
$$

where $\boldsymbol{k}^{\prime}:=\left(k_{1}^{\prime}, k_{2}^{\prime}, k_{3}^{\prime}\right)$ with $k_{i}^{\prime}:=2 \pi k_{i} / L_{i}$. For $\sigma>0$ fixed, the Gevrey norm $\|\cdot\|_{\sigma}$ is defined as

$$
\|W\|_{\sigma}^{2}:=\sum_{\boldsymbol{k} \in \mathbb{Z}^{3}} \mathrm{e}^{2 \sigma|\boldsymbol{k}|}\left|W_{\boldsymbol{k}}\right|^{2} .
$$

It follows from this that if $\|W\|_{\sigma}<\infty,\left|W_{\boldsymbol{k}}\right|$ decays exponentially fast in $|\boldsymbol{k}|$; this fact will play an important role below.

The following result on the OPEs is proved in [14] (this has been extended to all time in [12, but the version here is more useful for our present need):

Lemma 1. Let $W_{0}=\left(u_{0}, v_{0}, \rho_{0}\right)$ be such that $\left|\nabla_{3} W_{0}\right|=C_{1,0}<\infty$ and suppose that $\left|\boldsymbol{\nabla}_{3} f_{\boldsymbol{v}}\right|+\left|\boldsymbol{\nabla}_{3} f_{\rho}\right|=C_{f}<\infty$. Then for any $C_{1}>C_{1,0}$ there exists a $T_{1}\left(C_{1,0}, C_{f}, C_{1} ; \sigma, \mu, \mathcal{M}\right)>0$ such that for $t \in\left[0, T_{1}\right]$,

$$
\left|\nabla_{3} W(t)\right| \leq C_{1} .
$$


Now let $\sigma>0$ be fixed, and let $W_{0}$ be such that $\left\|\Delta_{3} W_{0}\right\|_{\sigma}=C_{\sigma, 0}<\infty$; in addition, suppose that $\left\|\nabla_{3} f_{\boldsymbol{v}}\right\|_{\sigma}+\left\|\nabla_{3} f_{\rho}\right\|_{\sigma}=C_{f}^{\prime}<\infty$. Then for any $C_{\sigma}>C_{\sigma, 0}$, there exists a $T_{\sigma}\left(C_{\sigma, 0}, C_{f}^{\prime}, C_{\sigma} ; \sigma, \mu, \mathcal{M}\right)>0$ such that for $t \in\left[0, T_{\sigma}\right]$,

$$
\left\|\Delta_{3} W(t)\right\|_{\sigma} \leq C_{\sigma}
$$

We stress that $T_{1}$ and $T_{\sigma}$ are independent of $\varepsilon$. (Here and henceforth, all constants are understood to be positive.)

Given a fixed $\kappa>0$, we define a finite-dimensional truncation of $W$ as

$$
W^{<}(\boldsymbol{x}, t):=\left(\mathrm{P}^{<} W\right)(\boldsymbol{x}, t):=\sum_{|\boldsymbol{k}|<\kappa} W_{\boldsymbol{k}}(t) \mathrm{e}^{\mathrm{i} \boldsymbol{k}^{\prime} \cdot \boldsymbol{x}},
$$

and $W^{>}:=W-W^{<}$. It is clear from this definition that the projection $\mathrm{P}^{<}$is orthogonal. For the low modes $W^{<}$we have the "reverse Poincaré inequality",

$$
\left|\nabla_{3} W^{<}\right|_{s} \leq c \kappa\left|W^{<}\right|_{s} .
$$

Here and throughout this article, $\|\cdot\|_{\sigma}$ denotes the Gevrey norm (2.2) and $|\cdot|_{s}$ denotes the usual Sobolev $H^{s}$ norm; when no confusion may arise, we will often write simply $|\cdot|$ for the $L^{2}$ norm $|\cdot|_{0}$. If $\|W\|_{\sigma}<\infty$, the exponential decay of the Fourier coefficients $W_{\boldsymbol{k}}$ allows us to bound the high modes $W^{>}$in $L^{2}$ as

$$
\left|W^{>}\right|_{0} \leq C_{0} \mathrm{e}^{-\sigma \kappa}\|W\|_{\sigma}
$$

or, more generally in $H^{s}$,

$$
\left|W^{>}\right|_{s} \leq C_{s} \kappa^{s} \mathrm{e}^{-\sigma \kappa}\|W\|_{\sigma} .
$$

We can now state our main result:

Theorem 1. Let $\sigma \in(0,1)$ be fixed. Suppose that the initial data $W_{0}=\left(u_{0}, v_{0}, \rho_{0}\right)$ satisfies

$$
\left\|\nabla_{3}^{3} W_{0}\right\|_{\sigma}<\infty
$$

and the forcing satisfies

$$
\left\|\nabla_{3}^{3} f_{\boldsymbol{v}}\right\|_{\sigma}+\left\|\nabla_{3}^{3} f_{\rho}\right\|_{\sigma}<\infty .
$$

Then there exists an $\varepsilon_{0}\left(\left\|\nabla_{3}^{3} W_{0}\right\|_{\sigma},\left\|\nabla_{3}^{3} f\right\|_{\sigma} ; \sigma, \mu, \mathcal{M}\right)>0$, and a $\tilde{q}$ (which is finite dimensional) evolving according to

$$
\partial_{t} \tilde{q}+\mathrm{P}^{<}\left[\boldsymbol{\nabla}^{\perp} \cdot\left(\boldsymbol{u}^{*} \cdot \boldsymbol{\nabla} \boldsymbol{v}^{*}\right)-\partial_{z}\left(\boldsymbol{u}^{*} \cdot \boldsymbol{\nabla} \rho^{*}\right)\right]=\mu \Delta_{3} \tilde{q}+f_{q}^{<},
$$

where $\boldsymbol{u}^{*}=\boldsymbol{u}^{*}(\tilde{q} ; \varepsilon)$ and $\rho^{*}=\rho^{*}(\tilde{q} ; \varepsilon)$ are constructed in the proof below, such that if $\varepsilon \in\left(0, \varepsilon_{0}\right)$ and the initial data satisfies

$$
\left|\boldsymbol{v}_{0}-\boldsymbol{v}^{*}(\tilde{q}(0) ; \varepsilon)\right|_{0}^{2}+\left|\rho_{0}-\rho^{*}(\tilde{q}(0) ; \varepsilon)\right|_{0}^{2} \leq C_{\text {id }} \exp \left(-2 \sigma / \varepsilon^{1 / 4}\right),
$$

for some constant $C_{\mathrm{id}}$ (independent of $\varepsilon$ and $W_{0}$ ) then a similar estimate,

$$
\left|\boldsymbol{v}(t)-\boldsymbol{v}^{*}(\tilde{q}(t) ; \varepsilon)\right|_{0}^{2}+\left|\rho(t)-\rho^{*}(\tilde{q}(t) ; \varepsilon)\right|_{0}^{2} \leq 4 C_{\mathrm{id}} \exp \left(-2 \sigma / \varepsilon^{1 / 4}\right),
$$

holds for $t \in\left[0, T_{*}\right]$ where $T_{*}=T_{*}\left(\left\|\nabla_{3}^{3} W_{0}\right\|_{\sigma},\left\|\nabla_{3}^{3} f\right\|_{\sigma} ; \sigma, \mu, \mathcal{M}\right)$.

\section{Remarks.}

1. The $\boldsymbol{v}^{*}$ and $\rho^{*}$ are the "exponential order" analogues of the (leading-order) quasi-geostrophic $\boldsymbol{v}^{\mathrm{g}}$ and $\rho^{\mathrm{g}}$. Unlike the latter, which is infinite-dimensional, our higher-order approximations are based on a finite-dimensional variable and depend on Gevrey regularity of the parent system (1.1). 
2. The sharpness of our bound (2.13) is unclear, but an explicit example constructed in [17] for a closely related problem suggests that one cannot do better than $\exp (-c / \varepsilon)$.

3. Although in this article we only treat the primitive equations for the ocean (1.1), the method described here should be applicable to many PDEs with a small parameter for which a Gevrey regularity result can be proved.

\section{Proof of Theorem 1}

Our approach is inspired by [11, which is an averaging-type result on singlefrequency infinite-dimensional systems, and by [5, App. B], which considers a finitedimensional problem. Our present problem is infinite-dimensional with an infinite number of frequencies, so small denominator problems would appear in an extension of [11] to this case. However, by considering the singular perturbation problem (hence the requirement for special initial data) in this article, we can avoid the small denominator problem.

The plan of the proof is as follows:

* We will build up our $n$ th-order approximation $W^{n}$ as:

$$
\begin{aligned}
& W=W^{<}+W^{>} \\
& W^{<}=W^{n}+\hat{W}
\end{aligned}
$$

The high modes $W^{>}$are exponentially small thanks to the Gevrey regularity of $W$; (2.8) then implies that they are exponentially small in any Sobolev norm. The low modes $W^{<}$(which are finite-dimensional) can then be approximated by $W^{n}$, which is constructed such that $\hat{W}$ is exponentially small in $\varepsilon$. The total error $W-W^{n}=W^{>}+\hat{W}$ is then also exponentially small. Like $W^{<}$, the approximate solution $W^{n}=\left(\boldsymbol{v}^{n}, \rho^{n}\right)$ is finite dimensional,

$$
W^{n}(\boldsymbol{x}, t)=\sum_{|\boldsymbol{k}|<\kappa} W_{\boldsymbol{k}}^{n}(t) \mathrm{e}^{\mathrm{i} \boldsymbol{k} \cdot \boldsymbol{x}} .
$$

* It is "slaved" to the slow variable $\tilde{q}$ in the following manner:

$$
\begin{aligned}
& \boldsymbol{v}^{n}=\overline{\boldsymbol{V}}^{n}+\nabla^{\perp} \psi^{n}+\nabla \mathrm{X}^{n} \quad \text { with } \quad \Delta_{3} \psi^{n}=\tilde{q}+\partial_{z z} \Phi^{n} \\
& \rho^{n}=\Delta_{3}^{-1}\left[\Delta_{2} \partial_{z} \Phi^{n}-\partial_{z} \tilde{q}\right],
\end{aligned}
$$

where $\overline{\boldsymbol{V}}(\tilde{q} ; \varepsilon), \mathrm{X}^{n}(\tilde{q} ; \varepsilon)$ and $\Phi^{n}(\tilde{q} ; \varepsilon)$ are functions to be computed below. For a motivation of this "slaving ansatz", we refer the reader to [20]. Even when working with finite-dimensional systems, we shall keep the convenient PDE notation, remembering that in this case we have the reverse Poincaré inequality (2.6). We note for future reference

$$
w^{n}=-\int_{0}^{z} \Delta_{2} \mathrm{X}^{n}(\tilde{q} ; \varepsilon) \mathrm{d} z^{\prime} .
$$

* The (truncated) potential vorticity $\tilde{q}$ evolves according to our "higher-order quasigeostrophic equations" $\left(\mathrm{QGE}^{n}\right)$,

$$
\partial_{t} \tilde{q}+\mathrm{P}^{<}\left[\boldsymbol{\nabla}^{\perp} \cdot\left(\boldsymbol{u}^{n} \cdot \boldsymbol{\nabla} \boldsymbol{v}^{n}\right)-\partial_{z}\left(\boldsymbol{u}^{n} \cdot \nabla \rho^{n}\right)\right]=\mu \Delta_{3} \tilde{q}+f_{q}^{<},
$$

where $\boldsymbol{u}^{n}=\left(\boldsymbol{v}^{n}, w^{n}\right)$ and $\rho^{n}$ are determined in terms of $\left(\tilde{q}, \overline{\boldsymbol{V}}^{n}, \mathrm{X}^{n}, \Phi^{n}\right)$ by (3.4)(3.6). 
* In turn, $\left(\overline{\boldsymbol{V}}^{n}, \mathrm{X}^{n}, \Phi^{n}\right)$ are computed using the iteration (3.11)-(3.13). If this iteration were convergent, say to $\left(\overline{\boldsymbol{V}}^{\infty}, \mathrm{X}^{\infty}, \Phi^{\infty}\right)$, and if we let $\tilde{q}$ be the solution of

$$
\partial_{t} \tilde{q}+\mathrm{P}^{<}\left[\boldsymbol{\nabla}^{\perp} \cdot\left(\boldsymbol{u}^{\infty} \cdot \boldsymbol{\nabla} \boldsymbol{v}^{\infty}\right)-\partial_{z}\left(\boldsymbol{u}^{\infty} \cdot \boldsymbol{\nabla} \rho^{\infty}\right)\right]=\mu \Delta_{3} \tilde{q}+f_{q}^{<},
$$

where $\boldsymbol{u}^{\infty}$ and $\rho^{\infty}$ are determined from $\left(\overline{\boldsymbol{V}}^{\infty}, \mathrm{X}^{\infty}, \Phi^{\infty}\right)$ as before, then $\left(\boldsymbol{v}^{\infty}, \rho^{\infty}\right)$ would be an exact solution of the OPEs. As is often the case in this type of problems (see 10] and 17] for closely related problems), this iteration is asymptotic rather than convergent, so we have to end the iteration at some $n=n_{*}$. It is shown below that for $n=0, \cdots, n_{*}=\left\lfloor\eta / \varepsilon^{1 / 4}\right\rfloor$ : (i) the slaved variables $\left(\overline{\boldsymbol{V}}^{n}, \mathrm{X}^{n}, \Phi^{n}\right)$ are bounded [see (3.39) below], and (ii) the $\mathrm{QGE}^{n}$ has an error of order $\varepsilon^{n}$ locally in time [see (3.56) below].

* We then show that with $\overline{\boldsymbol{v}}^{n}=\overline{\boldsymbol{V}}^{n}(\tilde{q} ; \varepsilon), \chi^{n}=\mathrm{X}^{n}(\tilde{q} ; \varepsilon)$ and $\phi^{n}=\Phi^{n}(\tilde{q} ; \varepsilon)$ the solution of (3.7) is bounded for $0 \leq t \leq T_{0}$ with $T_{0}$ independent of $\varepsilon$. (One can in fact show that the solution is bounded for all time, but we shall not do so here.)

* Finally, we obtain bounds for $\hat{W}$ using the usual Gronwall-type argument. This proves the main theorem since $W^{>}$is exponentially small by Lemma 1

Following a common practice, we write $c$ for a generic constant which may not be the same each time it appears; the more important constants are numbered: $c_{1}$, $c_{2}$, etc. Unless otherwise indicated, these constants (assumed positive) may depend on $s, \sigma$ and domain size $\left(L_{i}\right)$, but not on $\varepsilon, \kappa, n, \mu$ or the initial data.

3.1. Construction of $\mathbf{Q G E}{ }^{n}$. Let $\overline{\boldsymbol{V}}^{0}=0, \mathrm{X}^{0}=0$ and $\Phi^{0}=0$. From (3.4)-(3.5) we then have

$$
\boldsymbol{v}^{0}=\nabla^{\perp} \Delta_{3}^{-1} \tilde{q} \quad \text { and } \quad \rho^{0}=-\partial_{z} \Delta_{3}^{-1} \tilde{q} .
$$

Putting these in (3.7) and using the fact that $\boldsymbol{\nabla}^{\perp} \cdot\left(\boldsymbol{v}^{0} \cdot \boldsymbol{\nabla} \boldsymbol{v}^{0}\right)-\partial_{z}\left(\boldsymbol{v}^{0} \cdot \boldsymbol{\nabla} \rho^{0}\right)=\boldsymbol{v}^{0} \cdot \boldsymbol{\nabla} \tilde{q}$, we find

$$
\partial_{t} \tilde{q}+\mathrm{P}^{<}\left[\boldsymbol{v}^{0} \cdot \nabla \tilde{q}\right]=\mu \Delta_{3} \tilde{q}+f_{q}^{<},
$$

which is the quasi-geostrophic equation (1.4)-(1.5) for the truncated variable $\tilde{q}$. This is our zeroth-order (truncated) quasi-geostrophic model, $\mathrm{QGE}^{0}$.

For $n \geq 1$, we define $\overline{\boldsymbol{V}}^{n}(\tilde{q} ; \varepsilon), \mathrm{X}^{n}(\tilde{q} ; \varepsilon)$ and $\Phi^{n}(\tilde{q} ; \varepsilon)$ by the following iteration [cf. (1.14)]

$$
\begin{aligned}
&-\frac{1}{\varepsilon}\left(\overline{\boldsymbol{V}}_{z}^{n+1}\right)^{\perp}=\mathrm{P}^{<}\left\{\left(\mathrm{D} \overline{\boldsymbol{V}}_{z}^{n}\right) \mathcal{G}^{<}\left(\tilde{q}, \overline{\boldsymbol{V}}^{n}, \mathrm{X}^{n}, \Phi^{n}\right)+\partial_{z}\left(\overline{w^{n} \boldsymbol{v}_{z}^{n}}\right)\right\}-\mu \partial_{z z} \overline{\boldsymbol{V}}_{z}^{n}-\partial_{z} \bar{f}_{\boldsymbol{v}} \\
& \frac{1}{\varepsilon} \Delta_{3} \Phi^{n+1}=\mathrm{P}^{<}\left\{\left(\mathrm{D} \Delta_{3} \mathrm{X}^{n}\right) \mathcal{G}^{<}\left(\tilde{q}, \overline{\boldsymbol{V}}^{n}, \mathrm{X}^{n}, \Phi^{n}\right)\right. \\
&\left.\quad+\Delta_{3} \Delta_{2}^{-1} \boldsymbol{\nabla} \cdot\left(\boldsymbol{u}^{n} \cdot \boldsymbol{\nabla} \boldsymbol{v}^{n}\right)\right\}-\mu \Delta_{3}^{2} \mathrm{X}^{n}-\Delta_{3} f_{\chi}^{<} \\
&-\frac{1}{\varepsilon} \Delta_{3} \mathrm{X}^{n+1}=\mathrm{P}^{<}\left\{\left(\mathrm{D} \Delta_{3} \Phi^{n}\right) \mathcal{G}^{<}\left(\tilde{q}, \overline{\boldsymbol{V}}^{n} \mathrm{X}^{n}, \Phi^{n}\right)\right. \\
&\left.\quad+\partial_{z z} \Delta_{2}^{-1} \boldsymbol{\nabla}^{\perp} \cdot\left(\boldsymbol{u}^{n} \cdot \boldsymbol{\nabla} \boldsymbol{v}^{n}\right)+\mathrm{P}_{z} \partial_{z}\left(\boldsymbol{u}^{n} \cdot \boldsymbol{\nabla} \rho^{n}\right)\right\}-\mu \Delta_{3} \Phi_{z z}^{n}-\partial_{z z} f_{\phi}^{<}
\end{aligned}
$$

where $\mathrm{D}$ denotes derivative (differential) with respect to $\tilde{q}$ and

$$
\begin{aligned}
\mathcal{G}^{<}\left(\tilde{q}, \overline{\boldsymbol{V}}^{n}, \mathrm{X}^{n}, \Phi^{n}\right) & =\mathrm{P}^{<}\left[\mu \Delta_{3} \tilde{q}+f_{q}-\nabla^{\perp} \cdot\left(\boldsymbol{u}^{n} \cdot \nabla \boldsymbol{v}^{n}\right)+\partial_{z}\left(\boldsymbol{u}^{n} \cdot \nabla \rho^{n}\right)\right] \\
& =: \hat{\mathcal{G}}\left(\tilde{q}, \overline{\boldsymbol{V}}^{n}, \mathrm{X}^{n}, \Phi^{n}\right)+\mu \Delta_{3} \tilde{q}+f_{q}^{<} .
\end{aligned}
$$


We recall that $\left(\boldsymbol{u}^{n}, \rho^{n}\right)$ are given in terms of $\left(\overline{\boldsymbol{V}}^{n}, \mathrm{X}^{n}, \Phi^{n}\right)$ by (3.4)-(3.6). This construction was presented, in general and formally, in [18, which also proposed the use of a series expansion.

Let $s>3 / 2$ be fixed (in the theorem we use $s=2$, but we keep the general $s$ in the proof), and consider $\tilde{q}$ fixed for now. We start by estimating the nonlinear terms in $\mathcal{G}^{<}\left(\tilde{q}, \overline{\boldsymbol{V}}^{n}, \mathrm{X}^{n}, \Phi^{n}\right)=: \mathcal{G}^{n}$ using (1.10) and (3.4)-(3.6): For $\partial_{z}\left(\boldsymbol{u}^{n} \cdot \boldsymbol{\nabla} \rho^{n}\right)$, we compute

$$
\begin{aligned}
\left|\boldsymbol{v}^{n}\right|_{s} & \leq c\left(\left|\overline{\boldsymbol{V}}^{n}\right|_{s}+\left|\boldsymbol{\nabla}_{2} \psi^{n}\right|_{s}+\left|\boldsymbol{\nabla}_{2} \chi^{n}\right|_{s}\right) \\
& \leq c\left(\left|\overline{\boldsymbol{V}}^{n}\right|_{s}+|\tilde{q}|_{s-1}+\left|\boldsymbol{\nabla}_{3} \Phi^{n}\right|_{s}+\left|\nabla_{3} \mathrm{X}^{n}\right|_{s}\right) \\
\left|\boldsymbol{v}_{z}^{n}\right|_{s} & \leq c\left(\left|\overline{\boldsymbol{V}}_{z}^{n}\right|_{s}+\left|\boldsymbol{\nabla}_{2} \psi_{z}^{n}\right|_{s}+\left|\boldsymbol{\nabla}_{2} \chi_{z}^{n}\right|_{s}\right) \\
& \leq c\left(\left|\overline{\boldsymbol{V}}_{z}^{n}\right|_{s}+|\tilde{q}|_{s}+\left|\Delta_{3} \Phi^{n}\right|_{s}+\left|\Delta_{3} \mathrm{X}^{n}\right|_{s}\right), \\
\left|w^{n}\right|_{s} & \leq c\left|w_{z}^{n}\right|_{s} \leq c\left|\Delta_{3} \mathrm{X}^{n}\right|_{s}
\end{aligned}
$$

From these we have

$$
\begin{aligned}
& c\left|\boldsymbol{u}^{n}\right|_{s} \leq\left|\overline{\boldsymbol{V}}^{n}\right|_{s}+|\tilde{q}|_{s-1}+\left|\Delta_{3} \mathrm{X}^{n}\right|_{s}+\left|\nabla_{3} \Phi^{n}\right|_{s} \\
& c\left|\boldsymbol{u}_{z}^{n}\right|_{s} \leq\left|\overline{\boldsymbol{V}}_{z}^{n}\right|_{s}+|\tilde{q}|_{s}+\left|\Delta_{3} \mathrm{X}^{n}\right|_{s}+\left|\Delta_{3} \Phi^{n}\right|_{s} .
\end{aligned}
$$

Putting these together with

$$
c\left|\nabla_{3} \rho^{n}\right|_{s} \leq|\tilde{q}|_{s}+\left|\Delta_{3} \Phi^{n}\right|_{s} \quad \text { and } \quad c\left|\nabla_{3} \rho_{z}^{n}\right|_{s} \leq|\tilde{q}|_{s+1}+\left|\Delta_{3} \Phi^{n}\right|_{s+1}
$$

gives us

$$
\begin{aligned}
& \left|\boldsymbol{u}_{z}^{n} \cdot \nabla \rho^{n}\right|_{s} \leq c\left(|\tilde{q}|_{s}+\left|\overline{\boldsymbol{V}}_{z}^{n}\right|_{s}+\left|\Delta_{3} \Phi^{n}\right|_{s}+\left|\Delta_{3} \mathrm{X}^{n}\right|_{s}\right)\left(|\tilde{q}|_{s}+\left|\Delta_{3} \Phi^{n}\right|_{s}\right) \\
& \left|\boldsymbol{u}^{n} \cdot \boldsymbol{\nabla} \rho_{z}^{n}\right|_{s} \leq c\left(|\tilde{q}|_{s-1}+\left|\overline{\boldsymbol{V}}^{n}\right|_{s}+\left|\nabla_{3} \Phi^{n}\right|_{s}+\left|\Delta_{3} \mathrm{X}^{n}\right|_{s}\right)\left(|\tilde{q}|_{s+1}+\left|\Delta_{3} \Phi^{n}\right|_{s+1}\right),
\end{aligned}
$$

and, upon the use of (2.6),

$$
\left|\partial_{z}\left(\boldsymbol{u}_{z}^{n} \cdot \nabla \rho^{n}\right)\right|_{s} \leq c \kappa\left(|\tilde{q}|_{s}+\left|\overline{\boldsymbol{V}}_{z}^{n}\right|_{s}+\left|\Delta_{3} \Phi^{n}\right|_{s}+\left|\Delta_{3} \mathrm{X}^{n}\right|_{s}\right)^{2}
$$

For the other nonlinear term in $\mathcal{G}^{n}$,

$$
\boldsymbol{\nabla}^{\perp} \cdot\left(\boldsymbol{u}^{n} \cdot \boldsymbol{\nabla} \boldsymbol{v}^{n}\right)=\boldsymbol{u}^{n} \cdot \boldsymbol{\nabla} \Delta_{2} \psi^{n}+\left(\boldsymbol{\nabla}^{\perp} \boldsymbol{u}^{n}\right): \nabla_{3}\left(\overline{\boldsymbol{V}}^{n}+\nabla^{\perp} \psi^{n}+\boldsymbol{\nabla} \mathrm{X}^{n}\right)
$$

we need in addition

$$
\begin{aligned}
\left|\nabla_{2} \boldsymbol{u}^{n}\right|_{s} & \leq c\left(\left|\Delta_{2} \psi^{n}\right|_{s}+\left|\Delta_{2} \mathrm{X}^{n}\right|_{s}+\left|\nabla_{2} w^{n}\right|_{s}\right) \\
& \leq c\left(|\tilde{q}|_{s}+\left|\Delta_{3} \mathrm{X}^{n}\right|_{s+1}+\left|\Delta_{3} \Phi^{n}\right|_{s}\right), \\
\left|\nabla_{3} \boldsymbol{v}^{n}\right|_{s} & \leq c\left(\left|\overline{\boldsymbol{V}}_{z}^{n}\right|_{s}+\left|\Delta_{3} \psi^{n}\right|_{s}+\left|\Delta_{3} \mathrm{X}^{n}\right|_{s}\right) \\
& \leq c\left(|\tilde{q}|_{s}+\left|\overline{\boldsymbol{V}}_{z}^{n}\right|_{s}+\left|\Delta_{3} \mathrm{X}^{n}\right|_{s}+\left|\Delta_{3} \Phi^{n}\right|_{s}\right),
\end{aligned}
$$

and

$$
c\left|\nabla_{3} \Delta_{2} \psi^{n}\right|_{s} \leq|\tilde{q}|_{s+1}+\left|\Delta_{3} \Phi^{n}\right|_{s+1} .
$$

Putting these together and using (2.6) again, we find

$$
\left|\nabla^{\perp} \cdot\left(\boldsymbol{u}^{n} \cdot \nabla \boldsymbol{v}^{n}\right)\right|_{s} \leq c \kappa\left(|\tilde{q}|_{s}+\left|\overline{\boldsymbol{V}}_{z}^{n}\right|_{s}+\left|\Delta_{3} \Phi^{n}\right|_{s}+\left|\Delta_{3} \mathrm{X}^{n}\right|_{s}\right)^{2} .
$$

The linear terms are easily bounded, giving us

$$
\left|\mathcal{G}^{n}\right|_{s} \leq c_{1} \kappa\left(|\tilde{q}|_{s}+\left|\overline{\boldsymbol{V}}_{z}^{n}\right|_{s}+\left|\Delta_{3} \Phi^{n}\right|_{s}+\left|\Delta_{3} \mathrm{X}^{n}\right|_{s}\right)^{2}+\mu \kappa^{2}|\tilde{q}|_{s}+\left|f_{q}^{<}\right|_{s}
$$


Going next to the nonlinear terms in (3.11)-(3.13), we compute

$$
\left|\overline{w^{n} \boldsymbol{v}_{z}^{n}}\right|_{s} \leq c\left|w^{n}\right|_{s}\left|\nabla^{\perp} \psi_{z}^{n}+\nabla \mathrm{X}_{z}^{n}\right|_{s} \leq c \kappa\left|\Delta_{3} \mathrm{X}^{n}\right|_{s}\left(|\tilde{q}|_{s}+\left|\Delta_{3} \Phi^{n}\right|_{s}+\left|\Delta_{3} \mathrm{X}^{n}\right|_{s}\right) .
$$

The following estimates follow from our computation above:

$$
\begin{aligned}
\left|\Delta_{3} \Delta_{2}^{-1} \boldsymbol{\nabla} \cdot\left(\boldsymbol{u}^{n} \cdot \boldsymbol{\nabla} \boldsymbol{v}^{n}\right)\right|_{s} & \leq c \kappa^{3}\left(|\tilde{q}|_{s}+\left|\overline{\boldsymbol{V}}_{z}^{n}\right|_{s}+\left|\Delta_{3} \Phi^{n}\right|_{s}+\left|\Delta_{3} \mathrm{X}^{n}\right|_{s}\right)^{2}, \\
\left|\partial_{z z} \Delta_{2}^{-1} \boldsymbol{\nabla}^{\perp} \cdot\left(\boldsymbol{u}^{n} \cdot \boldsymbol{\nabla} \boldsymbol{v}^{n}\right)\right|_{s} & \leq c \kappa^{3}\left(|\tilde{q}|_{s}+\left|\overline{\boldsymbol{V}}_{z}^{n}\right|_{s}+\left|\Delta_{3} \Phi^{n}\right|_{s}+\left|\Delta_{3} \mathrm{X}^{n}\right|_{s}\right)^{2} .
\end{aligned}
$$

Using the relations (1.15), the forcing terms are bounded as

$$
\begin{array}{ll}
\left|f_{q}\right|_{s} \leq c\left(\left|f_{\boldsymbol{v}}\right|_{s+1}+\left|f_{\rho}\right|_{s+1}\right) & \left|\Delta_{3} f_{\chi}\right|_{s} \leq c\left|f_{\boldsymbol{v}}\right|_{s+3} \\
\left|\partial_{z} \bar{f}_{\boldsymbol{v}}\right|_{s} \leq\left|f_{\boldsymbol{v}}\right|_{s+1} & \left|\partial_{z z} f_{\phi}\right|_{s} \leq c\left(\left|f_{\boldsymbol{v}}\right|_{s+3}+\left|f_{\rho}\right|_{s+3}\right) .
\end{array}
$$

Moving on to terms such as $\mathrm{D}\left(\Delta_{3} \mathrm{X}^{n}\right) \mathcal{G}^{n}$ in (3.11) (3.13), we need a few definitions: Let $D_{\eta}(\tilde{q})$ be the complex $\eta$-neighbourhood of $\tilde{q}$ in $\mathrm{P}^{<} H^{s}(\mathcal{M})$. It can be defined by Fourier series as

$$
D_{\eta}(\tilde{q})=\left\{q^{\prime}: q^{\prime}(\boldsymbol{x})=\sum_{|\boldsymbol{k}|<\kappa} q_{\boldsymbol{k}}^{\prime} \mathrm{e}^{\mathrm{i} \boldsymbol{k} \cdot \boldsymbol{x}} \text { with } \sum_{|\boldsymbol{k}|<\kappa}|\boldsymbol{k}|^{2 s}\left|q_{\boldsymbol{k}}^{\prime}-\tilde{q}_{\boldsymbol{k}}\right|^{2}<\eta^{2}\right\} .
$$

We note that since $\tilde{q}(\boldsymbol{x})$ is real, the Fourier coefficients must satisfy $\tilde{q}_{-\boldsymbol{k}}=\overline{\tilde{q}}_{\boldsymbol{k}}$ where (here and only here) bar denotes complex conjugate; however, the Fourier coefficients $q_{\boldsymbol{k}}^{\prime}$ in (3.30) need not satisfy this constraint. Let $\delta>0$ be fixed. For any function $g$ of $\tilde{q}$, let

$$
|g|_{s ; n}:=\sup _{D_{\eta-n \delta}(\tilde{q})}|g(\tilde{q})|_{s}
$$

which is meaningful for $n=0, \cdots,\lfloor\eta / \delta\rfloor=: n_{*}$ when $D_{\eta-n \delta}(\tilde{q})$ is non-empty.

Let us also denote

$$
\left|U^{n}\right|_{s ; n}:=\left|\overline{\boldsymbol{V}}_{z}^{n}\right|_{s ; n}+\left|\Delta_{3} \Phi^{n}\right|_{s ; n}+\left|\Delta_{3} \mathrm{X}^{n}\right|_{s ; n}
$$

To bound $\mathrm{D}\left(\overline{\boldsymbol{V}}_{z}^{n}\right) \mathcal{G}^{n}, \mathrm{D}\left(\Delta_{3} \mathrm{X}^{n}\right) \mathcal{G}^{n}$ and $\mathrm{D}\left(\Delta_{3} \Phi^{n}\right) \mathcal{G}^{n}$, we use Cauchy's integral formula (cf. e.g., 9]): For $\varphi: \operatorname{cl} D_{\eta}(\tilde{q}) \rightarrow \mathbb{C}$ analytic with $\operatorname{cl} D_{\eta}(\tilde{q}) \subset \mathbb{C}^{l}$ and $\delta>0$, one can bound $\left|\varphi^{\prime}\right|$ in $\operatorname{cl} D_{\eta-\delta}(\tilde{q})$ by $|\varphi|$ in $\operatorname{cl} D_{\eta}(\tilde{q})$,

$$
\left|\varphi^{\prime} \cdot z\right|_{\operatorname{cl} D_{\eta-\delta}(\tilde{q})} \leq \frac{1}{\delta}|\varphi|_{\operatorname{cl} D_{\eta}(\tilde{q})}|z|_{\mathbb{C}^{l}}
$$

Now $\overline{\boldsymbol{V}}_{z}(\tilde{q} ; \varepsilon), \Delta_{3} \mathrm{X}^{n}(\tilde{q} ; \varepsilon)$ and $\Delta_{3} \Phi^{n}(\tilde{q} ; \varepsilon)$ are analytic functions of $\tilde{q}$, being polynomials in $\tilde{q}_{\boldsymbol{k}}$. Using this, we have

$$
\begin{aligned}
\left|\left(\mathrm{D} \Delta_{3} \Phi^{n}\right) \mathcal{G}^{n}\right|_{s, n+1} & \leq \frac{c}{\delta}\left|\Delta_{3} \Phi^{n}\right|_{s, n}\left|\mathcal{G}^{n}\right|_{s, n} \\
& \leq \frac{c}{\delta}\left|\Delta_{3} \Phi^{n}\right|_{s, n}\left\{\mu \kappa^{2}|\tilde{q}|_{s}+\left|f_{q}^{<}\right|_{s}+c \kappa^{2}\left(|\tilde{q}|_{s}+\left|U^{n}\right|_{s ; n}\right)^{2}\right\} .
\end{aligned}
$$

We have now bounded every term in (3.13): putting together (3.34), (3.29), (3.28) and (3.20), we find

$$
\begin{aligned}
\frac{1}{\varepsilon}\left|\Delta_{3} \mathrm{X}^{n+1}\right|_{s ; n+1} \leq & \mu \kappa^{2}\left|\Delta_{3} \Phi^{n}\right|_{s ; n}+\left|\partial_{z z} f_{\phi}^{<}\right|_{s}+c \kappa^{3}\left(|\tilde{q}|_{s}+\left|U^{n}\right|_{s ; n}\right)^{2} \\
& +\frac{c}{\delta}\left|\Delta_{3} \Phi^{n}\right|_{s ; n}\left\{\mu \kappa^{2}|\tilde{q}|_{s}+\left|f_{q}^{<}\right|_{s}+c \kappa^{2}\left(|\tilde{q}|_{s}+\left|U^{n}\right|_{s ; n}\right)^{2}\right\} .
\end{aligned}
$$


Similarly, we find for (3.12),

$$
\begin{aligned}
\frac{1}{\varepsilon}\left|\Delta_{3} \Phi^{n+1}\right|_{s ; n+1} \leq & \mu \kappa^{2}\left|\Delta_{3} \mathrm{X}^{n}\right|_{s ; n}+\left|\Delta_{3} f_{\chi}^{<}\right|_{s}+c \kappa^{3}\left(|\tilde{q}|_{s}+\left|U^{n}\right|_{s ; n}\right)^{2} \\
& +\frac{c}{\delta}\left|\Delta_{3} \Phi^{n}\right|_{s ; n}\left\{\mu \kappa^{2}|\tilde{q}|_{s}+\left|f_{q}^{<}\right|_{s}+c \kappa^{2}\left(|\tilde{q}|_{s}+\left|U^{n}\right|_{s ; n}\right)^{2}\right\}
\end{aligned}
$$

and for (3.11),

$$
\begin{aligned}
\frac{1}{\varepsilon}\left|\overline{\boldsymbol{V}}_{z}^{n+1}\right|_{s ; n+1} \leq & \mu \kappa^{2}\left|\overline{\boldsymbol{V}}_{z}^{n}\right|_{s ; n}+\left|\partial_{z} \bar{f}_{\boldsymbol{v}}\right|_{s}+c \kappa^{3}\left(|\tilde{q}|_{s}+\left|U^{n}\right|_{s ; n}\right)^{2} \\
& +\frac{c}{\delta}\left|\Delta_{3} \Phi^{n}\right|_{s ; n}\left\{\mu \kappa^{2}|\tilde{q}|_{s}+\left|f_{q}^{<}\right|_{s}+c \kappa^{2}\left(|\tilde{q}|_{s}+\left|U^{n}\right|_{s ; n}\right)^{2}\right\} .
\end{aligned}
$$

Putting these estimates together with (3.29), we find

$$
\begin{aligned}
\frac{1}{\varepsilon}\left|U^{n+1}\right|_{s ; n+1} \leq & \mu \kappa^{2}\left|U^{n}\right|_{s ; n}+\|f\|+c_{2} \kappa^{3}\left(|\tilde{q}|_{s}+\left|U^{n}\right|_{s ; n}\right)^{2} \\
& +\frac{c_{3}}{\delta}\left|U^{n}\right|_{s ; n}\left\{\mu \kappa^{2}|\tilde{q}|_{s}+\left|f_{q}^{<}\right|_{s}+c_{4} \kappa^{2}\left(|\tilde{q}|_{s}+\left|U^{n}\right|_{s ; n}\right)^{2}\right\},
\end{aligned}
$$

where $\|f\|:=\left|\left(f_{\boldsymbol{v}}^{<}, f_{\rho}^{<}\right)\right|_{s+3}$.

Now suppose that at iteration $n$, with $c_{1}=8 c_{2}$ we have

$$
\left|U^{n}\right|_{s ; n} \leq c_{1} \varepsilon^{1 / 4}\left(|\tilde{q}|_{s}^{2}+\|f\|\right) \text {. }
$$

Since $\overline{\boldsymbol{V}}^{0}=\mathrm{X}^{0}=\Phi^{0}=0$, this trivally holds for $n=0$. Taking $\delta=\varepsilon^{1 / 4}$ and $\kappa=\varepsilon^{-1 / 4}$, and substituting this into (3.38), we find that

$$
\left|U^{n+1}\right|_{s ; n+1} \leq c_{1} \varepsilon^{1 / 4}\left(|\tilde{q}|_{s}^{2}+\|f\|\right)
$$

provided that $\varepsilon$ satisfies the following constraints

$$
\begin{aligned}
\varepsilon \leq \min \left(1 /\left(16 \mu^{2}\right), 1 / c_{1}^{4},\left(c_{1} / 4\right)^{4 / 3}, 1 /\left(12 \mu c_{3}|\tilde{q}|_{s}\right)^{4}\right. \\
\left.1 /\left(12 c_{3}\|f\|\right)^{4 / 3}, 1 /\left(12 c_{3} c_{4}\left[2|\tilde{q}|_{s}+\|f\|\right]^{2}\right)^{4}\right) .
\end{aligned}
$$

By induction (3.39) can be seen to hold for $n=1, \cdots, n_{*}$. For future reference, we note that this restriction on $\varepsilon$ and (3.39) imply that

$$
|\tilde{q}|_{s}+\left|U^{n}\right|_{s} \leq c_{2}\left(|\tilde{q}|_{s}+\|f\|\right) \text {. }
$$

3.2. Local Error Bounds. Moving on to estimating the approximation error locally in time, we define

$$
\begin{aligned}
\mathcal{R}_{\overline{\boldsymbol{v}}}^{n}:= & \left(\mathrm{D} \overline{\boldsymbol{V}}_{z}^{n}\right) \mathcal{G}^{n}+\frac{1}{\varepsilon} \overline{\boldsymbol{V}}_{z}^{n}+\partial_{z}\left(\overline{w^{n} \boldsymbol{v}_{z}^{n}}\right)-\mu \partial_{z z} \overline{\boldsymbol{V}}_{z}^{n}-\partial_{z} \bar{f}_{\boldsymbol{v}}^{<} \\
= & \frac{1}{\varepsilon}\left[\overline{\boldsymbol{V}}_{z}^{n}-\overline{\boldsymbol{V}}_{z}^{n+1}\right] \\
\mathcal{R}_{\chi}^{n}:= & \left(\mathrm{D} \Delta_{3} \mathrm{X}^{n}\right) \mathcal{G}^{n}-\frac{1}{\varepsilon} \Delta_{3} \Phi^{n}+\Delta_{3} \Delta_{2}^{-1} \nabla \cdot\left(\boldsymbol{u}^{n} \cdot \boldsymbol{\nabla} \boldsymbol{v}^{n}\right)-\mu \Delta_{3}^{2} \mathrm{X}^{n}-\Delta_{3} f_{\chi}^{<} \\
= & \frac{1}{\varepsilon}\left[\Delta_{3} \Phi^{n+1}-\Delta_{3} \Phi^{n}\right] \\
\mathcal{R}_{\phi}^{n}:= & \left(\mathrm{D} \Phi_{z z}^{n}\right) \mathcal{G}^{n}+\frac{1}{\varepsilon} \Delta_{3} \mathrm{X}^{n}+\partial_{z z} \Delta_{2}^{-1} \nabla^{\perp} \cdot\left(\boldsymbol{u}^{n} \cdot \boldsymbol{\nabla} \boldsymbol{v}^{n}\right) \\
& \quad+\mathrm{P}_{z} \partial_{z}\left(\boldsymbol{u}^{n} \cdot \boldsymbol{\nabla} \rho^{n}\right)-\mu \Delta_{3} \Phi_{z z}^{n}-\partial_{z z} f_{\phi}^{<} \\
= & \frac{1}{\varepsilon}\left[\Delta_{3} \mathrm{X}^{n}-\Delta_{3} \mathrm{X}^{n+1}\right] .
\end{aligned}
$$


Since $\overline{\boldsymbol{V}}^{0}=0, \mathrm{X}^{0}=0$ and $\Phi^{0}=0$, we have

$$
\begin{aligned}
& \mathcal{R}_{\overline{\boldsymbol{v}}}^{0}=-\partial_{z} \bar{f}_{\boldsymbol{v}}^{<}, \\
& \mathcal{R}_{\chi}^{0}=\Delta_{3} \Delta_{2}^{-1} \nabla \cdot\left(\boldsymbol{v}^{0} \cdot \boldsymbol{\nabla} \boldsymbol{v}^{0}\right)-\Delta_{3} f_{\chi}^{<}, \\
& \mathcal{R}_{\phi}^{0}=\partial_{z z} \Delta_{2}^{-1} \nabla^{\perp} \cdot\left(\boldsymbol{v}^{0} \cdot \boldsymbol{\nabla} \boldsymbol{v}^{0}\right)+\mathrm{P}_{z} \partial_{z}\left(\boldsymbol{v}^{0} \cdot \nabla \rho^{0}\right)-\partial_{z z} f_{\phi}^{<},
\end{aligned}
$$

which leads to the estimate, denoting $\left|\mathcal{R}^{n}\right|_{s}:=\left|\mathcal{R}_{\overline{\boldsymbol{v}}}^{n}\right|_{s}+\left|\mathcal{R}_{\chi}^{n}\right|_{s}+\left|\mathcal{R}_{\phi}^{n}\right|_{s}$,

$$
\left|\mathcal{R}^{0}\right|_{s} \leq C \varepsilon^{-3 / 4}\left(|\tilde{q}|_{s}+\|f\|\right)^{2}
$$

For $n \geq 1$, let us denote $\delta \boldsymbol{v}^{n}:=\boldsymbol{v}^{n+1}-\boldsymbol{v}^{n}, \delta \rho^{n}:=\rho^{n+1}-\rho^{n}$, etc., and consider

$$
\begin{aligned}
\mathcal{R}_{\overline{\boldsymbol{v}}}^{n+1}= & \left(\mathrm{D} \overline{\boldsymbol{V}}_{z}^{n+1}\right) \mathcal{G}^{n+1}+\frac{1}{\varepsilon} \overline{\boldsymbol{V}}_{z}^{n+1}+\partial_{z}\left(\overline{w^{n+1} \boldsymbol{v}_{z}^{n+1}}\right)-\mu \partial_{z z} \overline{\boldsymbol{V}}_{z}^{n+1}-\partial_{z} \bar{f}_{\boldsymbol{v}}^{<} \\
= & \left(\mathrm{D} \overline{\boldsymbol{V}}_{z}^{n+1}\right) \delta \mathcal{G}^{n}-\varepsilon\left(\mathrm{D} \mathcal{R}_{\overline{\boldsymbol{v}}}^{n}\right) \mathcal{G}^{n} \\
& \quad+\partial_{z}\left(\overline{\delta w^{n} \overline{\boldsymbol{v}}_{z}^{n}}\right)+\partial_{z}\left(\overline{w^{n+1} \delta \boldsymbol{v}_{z}^{n}}\right)+\varepsilon \mu \partial_{z z} \mathcal{R}_{\overline{\boldsymbol{v}}}^{n}
\end{aligned}
$$

For the second equality, we have used the second equalities in (3.43)- (3.45) to write $\delta \overline{\boldsymbol{v}}^{n}$ in terms of $\mathcal{R}_{\overline{\boldsymbol{v}}}^{n}$, etc. To estimate these $\delta$-quantities, we compute

$$
\begin{aligned}
& \Delta_{3} \delta \rho^{n}=\Delta_{2}\left[\partial_{z} \delta \Phi^{n}\right] \\
\Rightarrow & \left|\nabla_{3} \delta \rho^{n}\right|_{s} \leq c\left|\Delta_{3} \delta \Phi^{n}\right|_{s} \leq \varepsilon c\left|\mathcal{R}_{\chi}^{n}\right|_{s} .
\end{aligned}
$$

Similarly, we find

$$
\begin{aligned}
\left|\partial_{z} \delta w^{n}\right|_{s} & =\left|\Delta_{2} \delta \mathrm{X}^{n}\right|_{s} \leq \varepsilon c\left|\mathcal{R}_{\phi}^{n}\right|_{s} \\
\left|\nabla_{3} \delta \boldsymbol{v}^{n}\right|_{s} & \leq c\left|\nabla_{3} \delta \overline{\boldsymbol{V}}^{n}\right|_{s}+\left|\Delta_{3} \delta \psi^{n}\right|_{s}+\left|\Delta_{3} \delta \mathrm{X}^{n}\right|_{s} \\
& \leq c\left(\left|\delta \overline{\boldsymbol{V}}_{z}^{n}\right|_{s}+\left|\partial_{z z} \delta \Phi^{n}\right|_{s}+\left|\Delta_{3} \delta \mathrm{X}^{n}\right|_{s}\right) \leq \varepsilon c\left|\mathcal{R}^{n}\right|_{s} \\
\Rightarrow \quad\left|\partial_{z} \delta \boldsymbol{u}^{n}\right|_{s} & \leq \varepsilon c\left|\mathcal{R}^{n}\right|_{s} .
\end{aligned}
$$

Estimating the terms in

$$
\begin{aligned}
\delta \mathcal{G}^{n}= & \partial_{z}\left(\delta \boldsymbol{u}^{n} \cdot \nabla \rho^{n}+\boldsymbol{u}^{n+1} \cdot \nabla \delta \rho^{n}\right)-\nabla^{\perp} \cdot\left(\delta \boldsymbol{u}^{n} \cdot \nabla \boldsymbol{v}^{n}+\boldsymbol{u}^{n+1} \cdot \boldsymbol{\nabla} \delta \boldsymbol{v}^{n}\right) \\
= & \delta \boldsymbol{u}_{z}^{n} \cdot \boldsymbol{\nabla} \rho^{n}+\delta \boldsymbol{u}^{n} \cdot \nabla \rho_{z}^{n}+\boldsymbol{u}_{z}^{n+1} \cdot \boldsymbol{\nabla} \delta \rho^{n}+\boldsymbol{u}^{n+1} \cdot \boldsymbol{\nabla} \delta \rho_{z}^{n} \\
& -\nabla^{\perp} \delta \boldsymbol{u}^{n}: \nabla \boldsymbol{v}^{n}-\delta \boldsymbol{u}^{n} \cdot \nabla \Delta_{2} \psi^{n}-\nabla^{\perp} \boldsymbol{u}^{n+1}: \boldsymbol{\nabla} \delta \boldsymbol{v}^{n}-\boldsymbol{u}^{n+1} \cdot \nabla \Delta_{2} \delta \psi^{n}
\end{aligned}
$$

using what we have computed above as

$$
\begin{aligned}
c\left|\delta \mathcal{G}^{n}\right|_{s} & \leq \varepsilon\left|\mathcal{R}^{n}\right|_{s}\left(|\tilde{q}|_{s}+\left|U^{n}\right|_{s}\right)+\varepsilon \kappa\left|\mathcal{R}^{n}\right|_{s}\left(|\tilde{q}|_{s}+\left|U^{n}\right|_{s}\right)+\varepsilon\left(|\tilde{q}|_{s}+\left|U^{n}\right|_{s}\right)\left|\mathcal{R}^{n}\right|_{s} \\
& +\varepsilon \kappa\left(|\tilde{q}|_{s}+\left|U^{n}\right|_{s}\right)\left|\mathcal{R}^{n}\right|_{s}+\varepsilon \kappa\left|\mathcal{R}^{n}\right|_{s}\left(|\tilde{q}|_{s}+\left|U^{n}\right|_{s}\right)+\varepsilon\left|\mathcal{R}^{n}\right|_{s}\left(|\tilde{q}|_{s}+\left|U^{n}\right|_{s}\right) \\
& +\varepsilon \kappa\left(|\tilde{q}|_{s}+\left|U^{n}\right|_{s}\right)\left|\mathcal{R}^{n}\right|_{s}+\varepsilon \kappa\left(|\tilde{q}|_{s}+\left|U^{n}\right|_{s}\right)\left|\mathcal{R}^{n}\right|_{s},
\end{aligned}
$$

we obtain

$$
\left|\delta \mathcal{G}^{n}\right|_{s} \leq \varepsilon^{3 / 4} c\left|\mathcal{R}^{n}\right|_{s}\left(|\tilde{q}|_{s}+\left|U^{n}\right|_{s}\right) \leq \varepsilon^{3 / 4} c\left|\mathcal{R}^{n}\right|_{s}\left(|\tilde{q}|_{s}+\|f\|\right)
$$


Estimating $\left(\mathrm{D} \overline{\boldsymbol{V}}_{z}^{n+1}\right) \delta \mathcal{G}^{n}$ and $\left(\mathrm{D} \mathcal{R}_{\overline{\boldsymbol{v}}}^{n}\right) \mathcal{G}^{n}$ in (3.47) using Cauchy's formula as before and using (3.42), we find

$$
\begin{aligned}
& c\left|\mathcal{R}_{\overline{\boldsymbol{v}}}^{n+1}\right|_{s ; n+1} \leq \frac{1}{\delta}\left|\overline{\boldsymbol{V}}_{z}^{n}\right|_{s ; n}\left|\delta \mathcal{G}^{n}\right|_{s}+\frac{\varepsilon}{\delta}\left|\mathcal{R}_{\overline{\boldsymbol{v}}}^{n}\right|_{s ; n}\left|\mathcal{G}^{n}\right|_{s} \\
& +\left|\partial_{z}\left(\overline{\delta w^{n} \overline{\boldsymbol{v}}_{z}^{n}}\right)\right|_{s}+\left|\partial_{z}\left(\overline{w^{n+1} \delta \boldsymbol{v}_{z}^{n}}\right)\right|_{s}+\varepsilon \mu \kappa^{2}\left|\mathcal{R}_{\overline{\boldsymbol{v}}}^{n}\right|_{s ; n} \\
& \leq \frac{\varepsilon \kappa}{\delta}\left|\overline{\boldsymbol{V}}_{z}^{n}\right|_{s ; n}\left|\mathcal{R}^{n}\right|_{s}\left(|\tilde{q}|_{s}+\|f\|\right)+\frac{\varepsilon \kappa}{\delta}\left(|\tilde{q}|_{s}+\|f\|\right)^{2}\left|\mathcal{R}_{\overline{\boldsymbol{v}}}^{n}\right|_{s ; n} \\
& +\frac{\mu \varepsilon \kappa^{2}}{\delta}\left|\mathcal{R}_{\overline{\boldsymbol{v}}}^{n}\right|_{s ; n}|\tilde{q}|_{s}+\varepsilon\left|\mathcal{R}^{n}\right|\left(|\tilde{q}|_{s}+\|f\|\right)+\mu \varepsilon \kappa^{2}\left|\mathcal{R}^{n}\right|_{s} \\
& \left|\mathcal{R}_{\overline{\boldsymbol{v}}}^{n+1}\right|_{s ; n+1} \leq c_{5}^{\prime} \varepsilon^{1 / 4}\left(|\tilde{q}|_{s}+\|f\|+c_{6}(\mu)\right)^{2}\left|\mathcal{R}^{n}\right|_{s ; n}
\end{aligned}
$$

The estimates for $\mathcal{R}_{\chi}^{n}$ and $\mathcal{R}_{\phi}^{n}$ are identical in form, giving us

$$
\left|\mathcal{R}^{n+1}\right|_{s ; n+1} \leq c_{5} \varepsilon^{1 / 4}\left(|\tilde{q}|_{s}+\|f\|+c_{6}\right)^{2}\left|\mathcal{R}^{n}\right|_{s ; n}
$$

So $\left|\mathcal{R}^{n}\right|_{s ; n}$ is decreasing in $n$ for $\varepsilon$ sufficiently small. Remembering that this construction is valid for $n=0, \cdots, n_{*},\left|\mathcal{R}^{n}\right|_{s ; n}$ is therefore smallest for $n=n_{*}=$ $\eta \varepsilon^{-1 / 4}$. More precisely, when

$$
c_{5} \varepsilon^{1 / 4}\left(|\tilde{q}|_{s}+\|f\|+c_{6}\right)^{2} \leq K<1
$$

we have

$$
\begin{aligned}
\left|\mathcal{R}^{n_{*}}\right|_{s ; n_{*}} & \leq\left|\mathcal{R}^{0}\right|_{s ; 0}\left[c_{5} \varepsilon^{1 / 4}\left(|\tilde{q}|_{s}+\|f\|+c_{6}\right)^{2}\right]^{n_{*}} \\
& \leq\left|\mathcal{R}^{0}\right|_{s ; 0} \exp \left(\varepsilon^{-1 / 4} \eta \log K\right) \\
& \leq c\left(|\tilde{q}|_{s}+\|f\|\right)^{2} \varepsilon^{-3 / 4} \exp \left(\varepsilon^{-1 / 4} \eta \log K\right)
\end{aligned}
$$

which is exponentially small in $\varepsilon$ (the argument of the exponential is negative since $K<1$ ). In what follows we fix $K$, say $K=1 / 2$, and set $\eta=\sigma / \log 2$. Assuming in addition that $\varepsilon \leq \sigma / 10$, we can write $\varepsilon^{-3 / 4} \exp \left(-\sigma / \varepsilon^{1 / 4}\right) \leq c \exp \left(-\sigma / \varepsilon^{1 / 4}\right)$ and

$$
\left|\mathcal{R}^{n_{*}}\right|_{s ; n_{*}} \leq c\left(|\tilde{q}|_{s}+\|f\|\right)^{2} \exp \left(-\sigma / \varepsilon^{-1 / 4}\right) .
$$

Remarks. A similar result for a different model was obtained in [19. This result, which does not depend on Gevrey regularity (the model was inviscid), appears to be peculiar to the model in question and is only local in time (i.e. only up to this point in the present proof).

3.3. Regularity of $\mathbf{Q G E}{ }^{n}$. We now show that the solution $\tilde{q}(t)$ of

$$
\partial_{t} \tilde{q}+\mathrm{P}^{<}\left[\boldsymbol{\nabla}^{\perp} \cdot\left(\boldsymbol{u}^{*} \cdot \boldsymbol{\nabla} \boldsymbol{v}^{*}\right)-\partial_{z}\left(\boldsymbol{u}^{*} \cdot \boldsymbol{\nabla} \rho^{*}\right)\right]=\mu \Delta_{3} \tilde{q}+f_{q}^{<}
$$

where $\boldsymbol{u}^{*}=\boldsymbol{u}^{n_{*}}$ and $\rho^{*}=\rho^{n_{*}}$, is bounded independently of $\varepsilon$ for $t \in\left[0, T_{0}\right]$. To this end we write $\boldsymbol{u}^{*}=\boldsymbol{u}^{0}+\boldsymbol{u}^{\varepsilon}$ and $\rho^{*}=\rho^{0}+\rho^{\varepsilon}$ where $\boldsymbol{u}^{0}=\left(u^{0}, v^{0}, 0\right)$,

$$
\boldsymbol{v}^{0}=\nabla^{\perp} \Delta_{3}^{-1} \tilde{q} \quad \text { and } \quad \rho^{0}=-\partial_{z} \Delta_{3}^{-1} \tilde{q} .
$$

As a preparation, we note that (3.19) and (3.23) imply, with (3.39),

$$
\begin{aligned}
& \left|\nabla_{3} \rho^{\varepsilon}\right|_{s} \leq c\left|\Delta_{3} \Phi^{*}\right|_{s} \leq c \varepsilon^{1 / 4}\left(|\tilde{q}|_{s}^{2}+\|f\|\right) \\
& \left|\nabla_{3} \boldsymbol{v}^{\varepsilon}\right|_{s} \leq c\left(\left|\overline{\boldsymbol{V}}_{z}^{*}\right|_{s}+\left|\Delta_{3} \mathrm{X}^{*}\right|_{s}+\left|\Delta_{3} \Phi^{*}\right|_{s}\right) \leq c \varepsilon^{1 / 4}\left(|\tilde{q}|_{s}^{2}+\|f\|\right) .
\end{aligned}
$$


Noting that $\nabla^{\perp} \cdot\left(\boldsymbol{v}^{0} \cdot \nabla \boldsymbol{v}^{0}\right)-\partial_{z}\left(\boldsymbol{v}^{0} \cdot \nabla \rho^{0}\right)=\boldsymbol{v}^{0} \cdot \boldsymbol{\nabla} \tilde{q}$, we write (3.57) as

$$
\begin{gathered}
\partial_{t} \tilde{q}+\mathrm{P}^{<}\left[\boldsymbol{v}^{0} \cdot \boldsymbol{\nabla} \tilde{q}+\boldsymbol{\nabla}^{\perp} \cdot\left(\boldsymbol{u}^{\varepsilon} \cdot \boldsymbol{\nabla} \boldsymbol{v}^{0}\right)+\nabla^{\perp} \cdot\left(\boldsymbol{v}^{0} \cdot \boldsymbol{\nabla} \boldsymbol{v}^{\varepsilon}\right)+\nabla^{\perp} \cdot\left(\boldsymbol{u}^{\varepsilon} \cdot \boldsymbol{\nabla} \boldsymbol{v}^{\varepsilon}\right)\right. \\
\left.-\partial_{z}\left(\boldsymbol{u}^{\varepsilon} \cdot \boldsymbol{\nabla} \rho^{0}\right)-\partial_{z}\left(\boldsymbol{v}^{0} \cdot \boldsymbol{\nabla} \rho^{\varepsilon}\right)-\partial_{z}\left(\boldsymbol{u}^{\varepsilon} \cdot \boldsymbol{\nabla} \rho^{\varepsilon}\right)\right]=\mu \Delta_{3} \tilde{q}+f_{q}^{<} .
\end{gathered}
$$

We multiply this by $\Delta_{3}^{s} \tilde{q}$ in $L^{2}(\mathcal{M})$ and estimate the resulting terms as

$$
\begin{aligned}
\left|\left(\Delta_{3}^{s} \tilde{q}, \boldsymbol{v}^{0} \cdot \nabla \tilde{q}\right)\right| & \leq c|\tilde{q}|_{s}\left|\boldsymbol{v}^{0} \cdot \nabla \tilde{q}\right|_{s} \leq c|\tilde{q}|_{s}|\tilde{q}|_{s-1}|\tilde{q}|_{s+1} \\
& \leq \frac{\mu}{4}|\tilde{q}|_{s+1}^{2}+\frac{c}{\mu}|\tilde{q}|_{s-1}^{2}|\tilde{q}|_{s}^{2}
\end{aligned}
$$

and for the terms involving $\boldsymbol{v}$,

$$
\begin{aligned}
\left|\left(\Delta_{3}^{s} \tilde{q}, \nabla^{\perp} \cdot\left(\boldsymbol{u}^{\varepsilon} \cdot \boldsymbol{\nabla} \boldsymbol{v}^{0}\right)\right)\right| & \leq c|\tilde{q}|_{s}\left|\boldsymbol{\nabla}^{\perp} \cdot\left(\boldsymbol{u}^{\varepsilon} \cdot \boldsymbol{\nabla} \boldsymbol{v}^{0}\right)\right|_{s} \leq c|\tilde{q}|_{s} \kappa\left|\boldsymbol{u}^{\varepsilon} \cdot \nabla \boldsymbol{v}^{0}\right|_{s} \\
& \leq c|\tilde{q}|_{s} \kappa\left|\boldsymbol{u}^{\varepsilon}\right|_{s}\left|\boldsymbol{\nabla}_{3} \boldsymbol{v}^{0}\right|_{s} \leq c|\tilde{q}|_{s} \kappa \varepsilon^{1 / 4}\left(|\tilde{q}|_{s}^{2}+\|f\|\right)|\tilde{q}|_{s} \\
& \leq c|\tilde{q}|_{s}^{2}\left(|\tilde{q}|_{s}^{2}+\|f\|\right), \\
\left|\left(\Delta_{3}^{s} \tilde{q}, \nabla^{\perp} \cdot\left(\boldsymbol{v}^{0} \cdot \boldsymbol{\nabla} \boldsymbol{v}^{\varepsilon}\right)\right)\right| & \leq c|\tilde{q}|_{s}^{2}\left(|\tilde{q}|_{s}^{2}+\|f\|\right), \\
\left|\left(\Delta_{3}^{s} \tilde{q}, \nabla^{\perp} \cdot\left(\boldsymbol{u}^{\varepsilon} \cdot \boldsymbol{\nabla} \boldsymbol{v}^{\varepsilon}\right)\right)\right| & \leq c|\tilde{q}|_{s} \kappa\left|\boldsymbol{u}^{\varepsilon}\right|_{s}\left|\boldsymbol{\nabla}_{3} \boldsymbol{v}^{\varepsilon}\right|_{s} \\
& \leq c \varepsilon^{1 / 4}|\tilde{q}|_{s}\left(|\tilde{q}|_{s}^{2}+\|f\|\right) ;
\end{aligned}
$$

and for those terms involving $\rho$,

$$
\begin{aligned}
& \mid\left(\Delta_{3}^{s} \tilde{q},\left.\partial_{z}\left(\boldsymbol{u}^{\varepsilon} \cdot \nabla \rho^{0}\right)|\leq c| \tilde{q}\right|_{s} ^{2}\left(|\tilde{q}|_{s}^{2}+\|f\|\right),\right. \\
& \mid\left(\Delta_{3}^{s} \tilde{q},\left.\partial_{z}\left(\boldsymbol{v}^{0} \cdot \nabla \rho^{\varepsilon}\right)|\leq c| \tilde{q}\right|_{s} ^{2}\left(|\tilde{q}|_{s}^{2}+\|f\|\right),\right. \\
& \mid\left(\Delta_{3}^{s} \tilde{q},\left.\partial_{z}\left(\boldsymbol{u}^{\varepsilon} \cdot \nabla \rho^{\varepsilon}\right)\left|\leq c \varepsilon^{1 / 4}\right| \tilde{q}\right|_{s}\left(|\tilde{q}|_{s}^{2}+\|f\|\right)^{2} .\right.
\end{aligned}
$$

Putting these together, we have

$$
\frac{\mathrm{d}}{\mathrm{d} t}|\tilde{q}|_{s}^{2}+\mu|\tilde{q}|_{s+1}^{2} \leq c_{8}(\mu)|\tilde{q}|_{s}\left(|\tilde{q}|_{s}^{2}+\|f\|+c^{\prime}\right)^{2}+c^{\prime \prime}(\mu)\|f\|^{2}
$$

where $c_{8}$ and $c^{\prime}$ are independent of $\varepsilon$ if one assumes (3.41) and (3.54). Now let $Q=2|\tilde{q}(0)|_{s}+\|f\|$. Replacing $|\tilde{q}|_{s}$ by $Q$ in (3.41) and (3.54), we assume

$$
\begin{gathered}
\varepsilon \leq \varepsilon_{0}=\min \left(\sigma / 10,1 /\left(16 \mu^{2}\right), 1 / c_{1}^{4},\left(c_{1} / 4\right)^{4 / 3}, 1 /\left(12 \mu c_{3} Q\right)^{4},\right. \\
1 /\left[2 c_{5}\left(Q+\|f\|+c_{6}\right)\right]^{4}, 1 /\left(12 c_{3}\|f\|\right)^{4 / 3} \\
\left.1 /\left[12 c_{3} c_{4}(2 Q+\|f\|)^{2}\right]^{4}\right) .
\end{gathered}
$$

It now follows from (3.68) that there exists a $T_{0}\left(|\tilde{q}(0)|_{s},\|f\| ; \mu, s, \sigma, \mathcal{M}\right)>0$ such that for $t \in\left[0, T_{0}\right]$,

(3.70) $|\tilde{q}(t)|_{s} \leq 2|\tilde{q}(0)|_{s}+\|f\|$.

Remarks. It is clear from the foregoing that this result is also valid for any $n \leq n_{*}$. When $n=0$ (i.e. the classical quasi-geostrophic equation), one does not need the $\kappa$ cutoff to prove boundedness (cf. [3]), but this seems unavoidable for $n>0$. 
3.4. Long-time Error Bounds. Let $W^{*}=\left(\boldsymbol{v}^{*}, \rho^{*}\right)=\left(\boldsymbol{v}^{n_{*}}, \rho^{n_{*}}\right)$. We now seek to show that $\hat{W}=W^{<}-W^{*}$, if initially exponentially small, remains exponentially small over a timescale of order one. We use the usual Gronwall-type argument. 
Starting with the evolution equation for $\rho^{<}$,

$$
\begin{aligned}
\partial_{t} \rho^{<}-\frac{1}{\varepsilon} w^{<}+\mathrm{P}^{<}\left(\boldsymbol{u}^{<} \cdot \nabla \rho^{<}\right)= & \mu \Delta_{3} \rho^{<}+f_{\rho}^{<} \\
& -\mathrm{P}^{<}\left(\boldsymbol{u}^{<} \cdot \boldsymbol{\nabla} \rho^{>}+\boldsymbol{u}^{>} \cdot \nabla \rho^{<}+\boldsymbol{u}^{>} \cdot \nabla \rho^{>}\right) \\
= & : \mu \Delta_{3} \rho^{<}+f_{\rho}^{<}+\mathcal{F}_{\rho}^{>},
\end{aligned}
$$

we find that $\hat{\rho}=\rho^{*}-\rho^{<}$is governed by

$$
\partial_{t} \hat{\rho}-\frac{1}{\varepsilon} \hat{w}+\mathrm{P}^{<}\left(\hat{\boldsymbol{u}} \cdot \nabla \hat{\rho}+\hat{\boldsymbol{u}} \cdot \nabla \rho^{*}+\boldsymbol{u}^{*} \cdot \nabla \hat{\rho}\right)=\mathcal{S}_{\rho}^{*}+\mu \Delta_{3} \hat{\rho}+\mathcal{F}_{\rho}^{>},
$$

where

$$
\mathcal{S}_{\rho}^{*}=-\partial_{t} \rho^{*}+\frac{1}{\varepsilon} w^{*}-\mathrm{P}^{<}\left(\boldsymbol{u}^{*} \cdot \nabla \rho^{*}\right)+\mu \Delta_{3} \rho^{*}+f_{\rho}^{<} .
$$

Now $\mathcal{S}_{\rho}^{*}$ can be expressed in terms of $\mathcal{R}_{\phi}^{*}$ as follows:

$$
\begin{aligned}
-\Delta_{3} \partial_{z} \mathcal{S}_{\rho}^{*}= & \partial_{t}\left(\Delta_{2} \Phi_{z z}^{*}-\partial_{z z} \tilde{q}\right)-\frac{1}{\varepsilon} \Delta_{3} \partial_{z} w^{*}+\Delta_{3} \partial_{z}\left(\boldsymbol{u}^{*} \cdot \nabla \rho^{*}\right) \\
& -\mu \Delta_{3} \partial_{z} \rho^{*}-\Delta_{3} \partial_{z} f_{\rho}^{<} \\
= & \Delta_{2}\left(\mathrm{D} \Phi_{z z}^{*}\right) \mathcal{G}^{*}-\partial_{z z} \mathcal{G}^{*}+\frac{1}{\varepsilon} \Delta_{3} \Delta_{2} \mathrm{X}^{*}+\Delta_{3} \partial_{z}\left(\boldsymbol{u}^{*} \cdot \nabla \rho^{*}\right) \\
& \quad-\mu \Delta_{3} \partial_{z} \rho^{*}-\Delta_{3} \partial_{z} f_{\rho}^{<}
\end{aligned}
$$

which gives upon using the definitions (3.14) of $\mathcal{G}^{*}$ and (3.45) of $\mathcal{R}_{\phi}$

(3.75) $\Delta_{3} \partial_{z} \mathcal{S}_{\rho}^{*}=\Delta_{2} \mathcal{R}_{\phi}^{*}$.

Similarly, we find for $\hat{\boldsymbol{v}}$,

$$
\begin{aligned}
\partial_{t} \hat{\boldsymbol{v}}+\frac{1}{\varepsilon}\left[\hat{\boldsymbol{v}}^{\perp}+\nabla \hat{p}\right]+\mathrm{P}^{<}\left(\hat{\boldsymbol{u}} \cdot \boldsymbol{\nabla} \hat{\boldsymbol{v}}+\hat{\boldsymbol{u}} \cdot \boldsymbol{\nabla} \boldsymbol{v}^{*}\right. & \left.+\boldsymbol{u}^{*} \cdot \boldsymbol{\nabla} \hat{\boldsymbol{v}}\right) \\
& =\mu \Delta_{3} \hat{\boldsymbol{v}}+\mathcal{S}_{\boldsymbol{v}}^{*}+\mathcal{F}_{\boldsymbol{v}}^{>}
\end{aligned}
$$

where

$$
\mathcal{F}_{\boldsymbol{v}}^{>}=-\mathrm{P}^{<}\left(\boldsymbol{u}^{<} \cdot \boldsymbol{\nabla} \boldsymbol{v}^{>}+\boldsymbol{u}^{>} \cdot \nabla \boldsymbol{v}^{<}+\boldsymbol{u}^{>} \cdot \boldsymbol{\nabla} \boldsymbol{v}^{>}\right)
$$

and, following the computation leading to (3.75),

$$
\overline{\mathcal{S}}_{\boldsymbol{v}}^{*}=\mathcal{R}_{\overline{\boldsymbol{v}}}^{*}, \quad \Delta_{3} \boldsymbol{\nabla}^{\perp} \cdot \mathcal{S}_{\boldsymbol{v}}^{*}=\Delta_{2} \mathcal{R}_{\phi}^{*} \quad \text { and } \quad \Delta_{3} \boldsymbol{\nabla} \cdot \mathcal{S}_{\boldsymbol{v}}^{*}=\Delta_{2} \mathcal{R}_{\chi}^{*}
$$

Multiplying (3.76) by $\hat{\boldsymbol{v}}$ and (3.72) by $\hat{\rho}$ in $L^{2}(\mathcal{M})$, we find

$$
\begin{aligned}
& \frac{1}{2} \frac{\mathrm{d}}{\mathrm{d} t}\left(|\hat{\boldsymbol{v}}|^{2}+|\hat{\rho}|^{2}\right)+\mu\left(\left|\nabla_{3} \hat{\boldsymbol{v}}\right|^{2}+\left|\nabla_{3} \hat{\rho}\right|^{2}\right) \\
& \quad=-\left(\hat{\boldsymbol{v}}, \hat{\boldsymbol{u}} \cdot \boldsymbol{\nabla} \boldsymbol{v}^{*}\right)-\left(\hat{\rho}, \hat{\boldsymbol{u}} \cdot \boldsymbol{\nabla} \rho^{*}\right)+\left(\hat{\boldsymbol{v}}, \mathcal{S}_{\boldsymbol{v}}^{*}\right)+\left(\hat{\rho}, \mathcal{S}_{\rho}^{*}\right)+\left(\hat{\boldsymbol{v}}, \mathcal{F}_{\boldsymbol{v}}^{>}\right)+\left(\hat{\rho}, \mathcal{F}_{\rho}^{>}\right) .
\end{aligned}
$$

Noting that

$$
\begin{aligned}
& \left|\nabla_{3} \boldsymbol{v}^{*}\right|_{L^{\infty}(\mathcal{M})} \leq c\left|\nabla_{3} \boldsymbol{v}^{*}\right|_{s} \leq c\left(|\tilde{q}|_{s}+\left|U^{*}\right|_{s}\right) \leq c\left(|\tilde{q}|_{s}+\|f\|\right) \\
& \left|\nabla_{3} \rho^{*}\right|_{L^{\infty}(\mathcal{M})} \leq c\left|\nabla_{3} \rho^{*}\right|_{s} \leq c\left(|\tilde{q}|_{s}+\left|U^{*}\right|_{s}\right) \leq c\left(|\tilde{q}|_{s}+\|f\|\right),
\end{aligned}
$$

where we have used (3.42) for the last inequalities, we bound the first two terms on the rhs of (3.79) by

$$
\begin{aligned}
& \left|\left(\hat{\boldsymbol{v}}, \hat{\boldsymbol{u}} \cdot \nabla \boldsymbol{v}^{*}\right)\right| \leq c|\hat{\boldsymbol{v}}|\left|\nabla_{3} \hat{\boldsymbol{v}}\right|\left|\nabla_{3} \boldsymbol{v}^{*}\right|_{L^{\infty}(\mathcal{M})} \leq \frac{\mu}{6}\left|\nabla_{3} \hat{\boldsymbol{v}}\right|^{2}+\frac{c}{\mu}|\hat{\boldsymbol{v}}|^{2}\left(|\tilde{q}|_{s}^{2}+\|f\|^{2}\right), \\
& \left|\left(\hat{\rho}, \hat{\boldsymbol{u}} \cdot \nabla \rho^{*}\right)\right| \leq c|\hat{\rho}|\left|\nabla_{3} \hat{\boldsymbol{v}}\right|\left|\nabla_{3} \rho^{*}\right|_{L^{\infty}(\mathcal{M N})} \leq \frac{\mu}{6}\left|\nabla_{3} \hat{\boldsymbol{v}}\right|^{2}+\frac{c}{\mu}|\hat{\rho}|^{2}\left(|\tilde{q}|_{s}^{2}+\|f\|^{2}\right),
\end{aligned}
$$


and use the Cauchy-Schwarz inequality for the remaining terms. This gives us

$$
\mu \frac{\mathrm{d}}{\mathrm{d} t}\left(|\hat{\boldsymbol{v}}|^{2}+|\hat{\rho}|^{2}\right) \leq c\left(|\tilde{q}|_{s}^{2}+\|f\|^{2}\right)\left(|\hat{\boldsymbol{v}}|^{2}+|\hat{\rho}|^{2}\right)+c^{\prime}\left|\mathcal{S}^{*}\right|^{2}+c^{\prime \prime}\left|\mathcal{F}^{>}\right|^{2},
$$

where we have denoted $\left|\mathcal{S}^{*}\right|^{2}=\left|\mathcal{S}_{\boldsymbol{v}}^{*}\right|^{2}+\left|\mathcal{S}_{\rho}^{*}\right|^{2}$ and $\left|\mathcal{F}^{>}\right|^{2}=\left|\mathcal{F}_{\boldsymbol{v}}^{>}\right|^{2}+\left|\mathcal{F}_{\rho}^{>}\right|^{2}$.

By (3.70), we can replace $|\tilde{q}(t)|_{s}$ by $2|\tilde{q}(0)|_{s}+\|f\|$ for $t \in\left[0, T_{0}\right]$. Using (3.75) and (3.78) we can then bound

$$
\left|\mathcal{S}^{*}(t)\right|_{0} \leq c\left|\mathcal{R}^{*}\right|_{s} \leq c\left(|\tilde{q}(0)|^{2}+\|f\|^{2}\right) \exp \left(-\sigma / \varepsilon^{1 / 4}\right),
$$

also valid for $t \in\left[0, T_{0}\right]$.

The last term in (3.82) can be bounded as

$$
\begin{aligned}
\left|\mathcal{F}^{>}\right|_{0} & \leq c\left|\nabla_{3} W^{<}\right|\left|\nabla_{3} W^{>}\right| \leq c\left|\nabla_{3} W\right|\left|\nabla_{3} W^{>}\right| \\
& \leq c \exp \left(-\sigma / \varepsilon^{1 / 4}\right)\left|\nabla_{3} W\right|\left\|\Delta_{3} W\right\|_{\sigma} .
\end{aligned}
$$

We now take $C_{1}=2\left|\nabla_{3} W_{0}\right|$ and $C_{\sigma}=2\left\|\Delta_{3} W_{0}\right\|_{\sigma}$ in Lemma 1 Since $\left|\nabla_{3} W_{0}\right| \leq$ $c\left\|\Delta_{3} W_{0}\right\|_{\sigma}$ by our hypothesis (i.e. $\sigma>0$ and the rhs is finite), we have

$$
\left|\nabla_{3} W(t)\right| \leq 2\left|\nabla_{3} W_{0}\right| \quad \text { and } \quad\left\|\Delta_{3} W(t)\right\|_{\sigma} \leq 2\left\|\Delta_{3} W_{0}\right\|_{\sigma}
$$

for $t \in\left[0, T_{\sigma}^{\prime}\right]$ where $T_{\sigma}^{\prime}\left(\left\|\Delta_{3} W_{0}\right\|_{\sigma},\|f\| ; \sigma, \mu, \mathcal{M}\right)=\min \left(T_{1}, T_{\sigma}\right)$. Thus (3.84) becomes

$$
\left|\mathcal{F}^{>}(t)\right|_{0} \leq c \exp \left(-\sigma / \varepsilon^{1 / 4}\right)\left\|\Delta_{3} W_{0}\right\|_{\sigma}^{2}
$$

for $t \in\left[0, T_{\sigma}^{\prime}\right]$.

Finally, writing $y=|\hat{\boldsymbol{v}}|^{2}+|\hat{\rho}|^{2}$, we rewrite (3.82) as

$$
\frac{\mathrm{d} y}{\mathrm{~d} t} \leq M y+N
$$

where $M$ is bounded independently of $\varepsilon$,

$$
M(t)=\frac{c}{\mu}\left(|\tilde{q}(t)|_{s}^{2}+\|f\|^{2}\right) \leq \frac{2 c}{\mu}\left(|\tilde{q}(0)|_{s}^{2}+\|f\|^{2}\right) \leq \frac{c^{\prime}}{\mu}\left(\left\|\Delta_{3} q_{0}\right\|_{\sigma}^{2}+\|f\|^{2}\right)
$$

for $t \in\left[0, T_{0}\right]$. As for $N$, 3.83) and (3.86) give us

$$
\begin{aligned}
N(t) & =\frac{c}{\mu}\left(\left|\mathcal{S}^{*}(t)\right|^{2}+\left.\mathcal{F}^{>}(t)\right|^{2}\right) \\
& \leq c_{9}(\mu)\left[\left(\left|\nabla_{3}^{3} W_{0}\right|+\|f\|\right)^{2}+\left\|\Delta_{3} W_{0}\right\|_{\sigma}^{2}\right] \exp \left(-2 \sigma / \varepsilon^{1 / 4}\right),
\end{aligned}
$$

valid for $t \in\left[0, T_{\sigma}^{\prime \prime}\right]$ where $T_{\sigma}^{\prime \prime}\left(\left\|\nabla_{3}^{3} W_{0}\right\|_{\sigma},\|f\| ; \sigma, \mu, \mathcal{M}\right)=\min \left(T_{0}, T_{\sigma}^{\prime}\right)$.

Since $W^{>}$and $\hat{W}$ are $L^{2}(\mathcal{M})$-orthogonal, the hypothesis (2.12) implies that

$$
\left|\hat{W}_{0}\right|^{2}+\left|W_{0}^{>}\right|^{2} \leq C_{\text {id }} \exp \left(-2 \sigma / \varepsilon^{1 / 4}\right) .
$$

Lemma 1 and (2.7) then imply that there is a $T_{3}\left(\left\|\nabla_{3}^{3} W_{0}\right\|_{\sigma},\|f\| ; \sigma, \mu, \mathcal{M}\right)$ such that for $t \in\left[0, T_{3}\right]$,

$$
\left|W^{>}(t)\right|^{2} \leq 2 C_{\mathrm{id}} \exp \left(-2 \sigma / \varepsilon^{1 / 4}\right) .
$$

Integrating (3.87) and taking into account (3.88)-(3.90) give us

$$
|\hat{W}(t)|^{2} \leq 2 C_{\text {id }} \exp \left(-2 \sigma / \varepsilon^{1 / 4}\right)
$$

for $t \in\left[0, T_{4}\right]$ with $T_{4}=T_{4}\left(\left\|\nabla_{3}^{3} W_{0}\right\|_{\sigma},\|f\| ; \sigma, \mu, \mathcal{M}\right)$. It thus follows that

$$
\left|W^{>}(t)\right|^{2}+|\hat{W}(t)|^{2} \leq 4 C_{\text {id }} \exp \left(-2 \sigma / \varepsilon^{1 / 4}\right),
$$

for $t \in\left[0, \min \left(T_{3}, T_{4}\right)\right]$, which is precisely (2.13). 


\section{REFERENCES}

[1] J. S. Allen, Iterated geostrophic intermediate models, J. Phys. Ocean., 23 (1993), pp. 24472461.

[2] P. Bartello, Geostrophic adjustment and inverse cascades in rotating stratified turbulence, J. Atmos. Sci., 52 (1995), pp. 4410-4428.

[3] A. J. Bourgeois and J. T. Beale, Validity of the quasigeostrophic model for large-scale flow in the atmosphere and ocean, SIAM J. Math. Anal., 25 (1994), pp. 1023-1068.

[4] C. CAO And E. S. Titi, Global well-posedness of the three-dimensional viscous primitive equations of large scale ocean and atmosphere dynamics. arXiv:math.AP/0503028 1, 2005.

[5] C. J. CotTer, Model reduction for shallow water dynamics: balance, adiabatic invariance and subgrid modelling, $\mathrm{PhD}$ thesis, Imperial College, London, 2004.

[6] N. Ju, The global attractor for the solutions to the 3D viscous Primitive Equations, Discrete Cont. Dyn. Systems, Ser. A, (2006), to appear.

[7] G. M. Kobelkov, Existence of a solution "in whole" for the large-scale ocean dynamics equations, C. R. Acad. Sc. Paris, Sér. A, (2006), to appear.

[8] J.-L. Lions, R. Temam, And S. Wang, On the equations of the large-scale ocean, Nonlinearity, 5 (1992), pp. 1007-1053.

[9] P. Lochak And C. Meunier, Multiphase averaging for classical systems, Springer-Verlag, 1988.

[10] E. N. Lorenz, Attractor sets and quasi-geostrophic equilibrium, J. Atmos. Sci., 37 (1980), pp. $1685-1699$.

[11] K. Matthies, Time-averaging under fast periodic forcing of parabolic partial differential equations: exponential estimates, J. Diff. Eq., 174 (2001), pp. 133-180.

[12] M. Petcu, On the three dimensional primitive equations, Adv. Diff. Eq., (2006), to appear.

[13] M. Petcu, R. Temam, and D. Wirosoetisno, Renormalization group method applied to the primitive equation, J. Diff. Eq., 208 (2004), pp. 215-257.

[14] M. Petcu And D. Wirosoetisno, Sobolev and Gevrey regularity results for the primitive equations in 3 space dimensions, Applic. Anal., 84 (2005), pp. 769-788.

[15] R. Temam, Infinite-dimensional dynamical systems in mechanics and physics, 2nd ed., Springer-Verlag, 1997.

[16] R. Temam And D. Wirosoetisno, Long-time balance for the primitive equations of the ocean. Article in preparation, 2006.

[17] J. Vanneste And I. Yavneh, Exponentially small inertia-gravity waves and the breakdown of quasi-geostrophic balance, J. Atmos. Sci., 61 (2004), pp. 211-223.

[18] T. Warn, O. Bokhove, T. G. Shepherd, and G. K. Vallis, Rossby number expansions, slaving principles, and balance dynamics, Quart. J. Roy. Met. Soc., 121 (1995), pp. 723-739.

[19] D. Wirosoetisno, Exponentially accurate balanced dynamics, Adv. Diff. Eq., 9 (2004), pp. $177-196$.

[20] D. Wirosoetisno, T. G. Shepherd, and R. M. Temam, Free gravity waves and balance dynamics, J. Atmos. Sci., 59 (2002), pp. 3382-3398.

E-mail address: temam@indiana.edu

$U R L:$ http://mypage.iu.edu/ ${ }^{\text {temam }}$

E-mail address: djoko.wirosoetisno@durham.ac.uk

URL: http://www.maths.dur.ac.uk/ ${ }^{\text {dmaOdw }}$

Current address, Wirosoetisno: Department of Mathematical Sciences, University of Durham,

Durham, DH1 3LE, United Kingdom

The Institute for Scientific Computing and Applied Mathematics, Indiana University, Rawles Hall, Bloomington, IN 47405-7106, United States 